\title{
Augmenting Vehicle Localization by Cooperative Sensing of the Driving Environment: Insight on Data Association in Urban Traffic Scenarios
}

\author{
Mattia Brambilla, Student Member, IEEE, Monica Nicoli, Member, IEEE, Gloria Soatti, Francesco Deflorio
}

\begin{abstract}
Precise vehicle positioning is a key element for the development of Cooperative Intelligent Transport Systems (CITS). In this context, we present a distributed processing technique to augment the performance of conventional Global Navigation Satellite Systems (GNSS) exploiting Vehicle-to-anything (V2X) communication systems. We propose a method, referred to as Implicit Cooperative Positioning with Data Association (ICPDA), where the connected vehicles detect a set of passive features in the driving environment, solve the association task by pairing them with on-board sensor measurements and cooperatively localize the features to enhance the GNSS accuracy. We adopt a belief propagation algorithm to distribute the processing over the network, and solve both the data association and localization problems locally at vehicles. Numerical results on realistic traffic networks show that the ICP-DA method is able to significantly outperform the conventional GNSS. In particular, the analysis on a real urban road infrastructure highlights the robustness of the proposed method in real-life cases where the interactions among vehicles evolve over space and time according to traffic regulation mechanisms. Performances are investigated both in conventional traffic-light regulated scenarios and self-regulated environments (as representative of future automated driving scenarios) where vehicles autonomously cross the intersections taking gap-availability decisions for avoiding collisions. The analysis shows how the mutual coordination in platoons of vehicles eases the cooperation process and increases the positioning performance.
\end{abstract}

Index Terms-Cooperative localization, V2X communications, cooperative ITS, distributed Bayesian tracking, data association, traffic simulation, controlled arterials.

\section{INTRODUCTION}

$\mathbf{C}$ OOPERATIVE Intelligent Transportation Systems (CITS) rely on Vehicle-to-anything (V2X) communications to enable fast diffusion of sensor data and prompt reaction to any anomalous event detected in the driving environment. Existing V2X standards for driver assistance are based on a WiFi mode (i.e., IEEE 802.11p WAVE [1] and ETSI ITS-G5 [2], for US and EU respectively) or on cellular communications (i.e., the recently released 3GPP LTE C-V2X standard [3]), while new millimeter-wave technologies are emerging in the context of the fifth-generation $(5 \mathrm{G})$ mobile networks to cover higher levels of automation [4], [5]. These technologies enable cooperative perception and maneuvering functionalities by direct vehicle-to-vehicle (V2V) exchange of sensor data and mobility patterns between vehicles, paving the way for a new cooperative, connected and automated mobility [6][10]. By fast V2V interactions, vehicles are able to fuse local and remote maps of the driving environment, extending the perception range much beyond the immediate field of view, with huge benefits in safety [11]. They can also exchange the intended trajectories to synchronize to a common mobility pattern, forming high-density platoons and enhancing traffic efficiency [12].

In many C-ITS applications, such as cooperative maneuvering or vulnerable road-user discovery [13]-[16], a fundamental requirement is precise vehicle positioning. Global Navigation Satellite Systems (GNSSs), even when augmented by inertial sensors, differential corrections or multi-constellation receivers, cannot guarantee the required navigation performance in terms of availability and accuracy, especially in highly builtup areas, where the satellite signal is severely attenuated or even denied [17], [18]. To improve GNSS performance, both non-cooperative and cooperative localization techniques have been explored. Among the first ones, a relevant approach is Simultaneous Localization And Mapping (SLAM) [19], [20] where an ego vehicle builds a detailed map of the surroundings and integrates the GNSS information in the mapping process to enhance the localization accuracy [21], [22]. With this method, vehicles rely only on their own sensors, without any data fusion with other vehicles. On the other hand, Cooperative Positioning (CP) techniques [23]-[31] perform data sharing over V2X links to enrich the set of location information. Overviews on $\mathrm{CP}$ technologies, protocols and algorithms can be found in [32]-[34]. Most of the proposed methods rely on explicit inter-vehicle measurements (distance, velocity or angle) extracted from the received V2X radio signals, such as time-of-flight [35], received signal strength [36], angle of arrival [37] or Doppler shift of the carrier frequency [38]. These approaches, however, either require high-complexity processing, dedicated hardware or external infrastructure. Moreover, they need vehicles to extract explicit range measurements (e.g., time of flight or round trip time by unicast communications) from the $\mathrm{V} 2 \mathrm{~V}$ radio signals, whilst current V2X standards [1], [2] cannot provide such information as they rely on broadcast transmissions.

Recently, the Implicit Cooperative Positioning (ICP) technique has been proposed where vehicles use on-board sensing equipment to detect a common set of passive objects in the driving environment. They then share the data over the V2V links to cooperatively localize these features with high precision [39], [40]. The geo-localized objects serve as additional reference points for GNSS augmentation. ICP belongs to the class of multi-sensor multi-target tracking methods [41], [42], where non-cooperative objects (targets) are sensed by a network of cooperative mobile agents (sensors) and used 
for localization. With respect to other $\mathrm{CP}$ methods for vehicle localization, the advantage is that ICP relies on conventional sensing and $\mathrm{V} 2 \mathrm{~V}$ communication devices, without requiring any explicit inter-vehicle ranging procedure or complex infrastructure. Only the location statistics based on post-processing of standard automotive sensor measurements are exchanged between vehicles.

The ICP method developed in [39], [40] assumed perfect association between measurements and sensed objects. However, in real C-ITS scenarios, data association (DA) is a mandatory step to enable a consistent fusion of measurements provided by multiple sensors at different vehicles. The DA issue, in fact, consists of solving the uncertainty on the origin of measurements. A solution to the DA problem has been proposed in [43] for centralized tracking of passive targets in sensor networks with known sensor locations, using an efficient association algorithm [44]. A similar centralized approach has been developed in [45] for vehicular environments. These methods employ Particle Filtering (PF) to sample the location statistics, which are non-Gaussian due to the association uncertainty. Unfortunately, in highly dynamic C-ITS scenarios where both vehicles and features have to be tracked, a large number of particles is required to sample the vehicle/feature densities with enough accuracy and the computational burden becomes easily unfeasible. Moreover, these DA-enabled methods rely on a central unit for data gathering and processing, whereas low-complexity decentralized techniques are preferred in CITS scenarios. To the best of our knowledge, fully distributed DA-enabled CP solutions have not been proposed in the literature. Furthermore, an in-depth performance analysis in realistic environments with large numbers of vehicles/features and varying traffic conditions is crucial to highlight how the localization accuracy scales with the traffic demand and to validate the method robustness.

This paper aims to fill the above gap by providing a twofold contribution that can be summarized as: ( $i$ ) extension of the ICP method such that DA issue is integrated and resolved; (ii) validation in realistic urban traffic scenario. The two contributions are detailed in the following.

The first contribution is the extension of the ICP method [40] to integrate the DA task into the distributed framework for localization of features and vehicles. Differently from previous works, which rely either on centralized processing [45] or known sensor locations [43], the proposed method jointly solves the DA issue and the feature-vehicle localization by distributed processing at vehicles. The proposed ICP method with Data Association (ICP-DA) relies on two Belief Propagation Algorithms (BPA) [46]: one for data association (BPA-DA), and the other for localization of features and vehicles (BPA-L). At first, vehicles use their on-board sensing equipment (e.g., radar, lidar or camera) to detect a number of nearby features and individually run BPA-DA to solve the association problem. Then, they use BPA-L to combine the Vehicle-To-Feature (V2F) information with the local GNSS measurement, sharing the information with neighbors by $\mathrm{V} 2 \mathrm{~V}$ communications. For the implementation of the ICP-DA method, we design a PF algorithm which relies on a particle-based representation of the non-Gaussian vehicle/feature location beliefs, combined with a consensus algorithm [47] for distributed computation of feature-related information at vehicles. We also propose a low-complexity (LC) suboptimal method, referred to as ICP-DA-LC, based on a hard Maximum-A-Posteriori (MAP) Bayesian detection for feature-measurement pairing and Kalman Filtering (KF) with Gaussian message passing for cooperative localization. The proposed MAP-based solution applies a feature selection criterion to exclude ambiguous V2F measurements and reduce the impact of detection errors. A preliminary version of the proposed method has been presented in [48], focusing on lowcomplexity ICP-DA implementation and static features. Here the work is extended with the derivation of the optimal PFbased algorithm and the introduction of feature mobility to handle realistic C-ITS scenarios.

The second main contribution of this paper is the validation of the proposed technique in a realistic urban traffic scenario. The localization performance is investigated by simulating traffic flow along an urban arterial of the road network in Turin, Italy, including primary roads and several secondary junctions. With respect to other studies where the positioning precision is randomly and exogenously generated, as in [49], in this work the vehicle localization accuracy is considered as dependent on the traffic conditions over the roads and the related traffic control systems, that rule the spatial distribution of the vehicles and their mutual interactions. A key element is the control strategy implemented at road intersections which affects the traffic flow in terms of delays, queues and capacity at the nodes, as well as the density of vehicles along the arterials. Two different traffic scenarios are analyzed: a standard one, where traffic lights regulate the vehicles dynamics at intersections, and a vehicle self-regulated scenario (representative of higher levels of automation) where vehicles individually and autonomously decide if they can safely cross an intersection avoiding conflicts. The simulation of real-life traffic conditions is instrumental for the assessment of the potential benefits of the proposed cooperative methods in typical urban conditions. An important goal is to show the strengths and drawbacks of one method with respect to the other, with main focus on the localization performance in terms of accuracy and computational complexity, which are key points to be addressed in vehicular applications. Furthermore, we aim to demonstrate that a sub-meter accuracy can be reached by the proposed cooperative localization approach which thus enables new CITS services, such as turn-by-turn route guidance, including lane selection, or queue length monitoring for optimal tuning of traffic light control [50].

The paper is organized as follows. Sec. II introduces the CITS model. Sec. III explains the Bayesian ICP-DA approach for the joint association and localization problem, while Sec. IV presents the distributed implementations by the PF (ICPDA-PF) and low-complexity KF (ICP-DA-LC) approaches. The performance analysis is in Sec. V and VI for, respectively, simplified and realistic road networks. Finally, Sec. VII draws the concluding remarks.

Notation. Bold upper- and lower-case letters describe matrices and column vectors. Matrix transposition is indicated as $(\cdot)^{\mathrm{T}}, \mathbf{I}_{m}$ denotes the identity matrix of size $m$, while $\mathbf{0}_{m \times n}$ is 


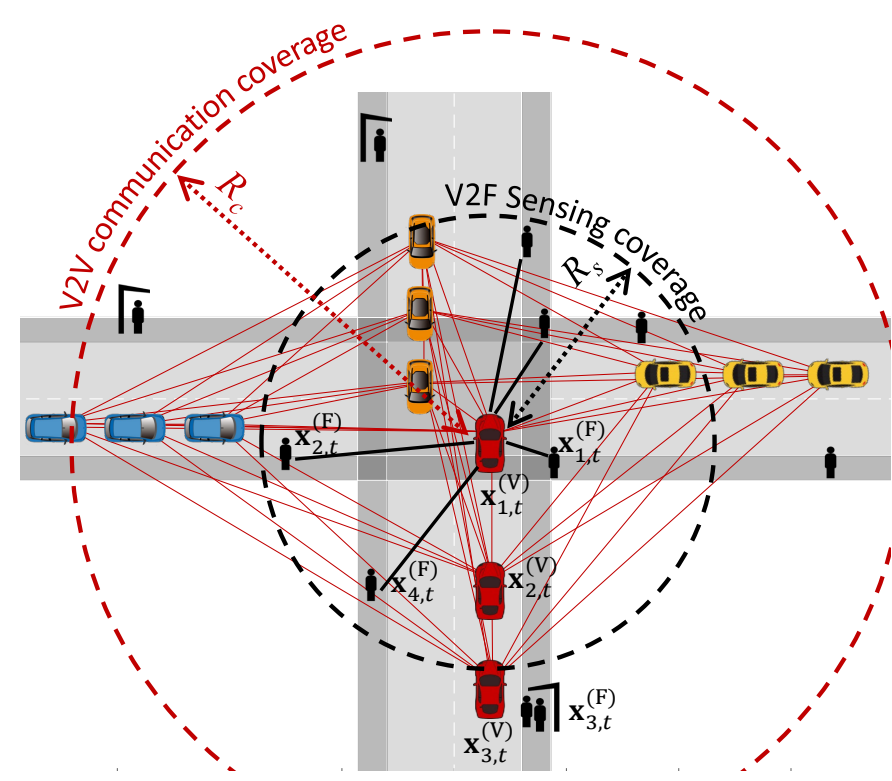

Fig. 1: Example of C-ITS scenario with $N_{v}=12$ connected vehicles and $N_{f}=10$ passive features. The red links indicate all the $\mathrm{V} 2 \mathrm{~V}$ connections, while the black ones represent the $\mathrm{V} 2 \mathrm{~F}$ connections of vehicle $i=1$. The communication and sensing ranges, $R_{c}$ and $R_{s}$, respectively, are referred to vehicle $i=1$.

a null matrix of size $m \times n$. $\mathbb{R}$ is the symbol of the set of real numbers. Lastly, operator $\|\cdot\|$ denotes the Euclidean norm.

\section{C-ITS MODEL}

We consider a C-ITS scenario where a set $\mathcal{V}=\left\{1, \ldots, N_{v}\right\}$ of $N_{v}$ interconnected vehicles is deployed over a two dimensional space as illustrated in Fig. 1. At time instant $t$, vehicle $i \in \mathcal{V}$ is characterized by the position $\mathbf{p}_{i, t}^{(\mathrm{V})}=\left[p_{x_{i, t}}^{(\mathrm{V})} p_{y_{i, t}}^{(\mathrm{V})}\right]^{\mathrm{T}} \in$ $\mathbb{R}^{2 \times 1}$ and velocity $\mathbf{v}_{i, t}^{(\mathrm{V})}=\left[v_{x_{i, t}}^{(\mathrm{V})} v_{y_{i, t}}^{(\mathrm{V})}\right]^{\mathrm{T}} \in \mathbb{R}^{2 \times 1}$, and it is able to exchange data with neighboring vehicles through $\mathrm{V} 2 \mathrm{~V}$ communications. The set of neighbors that directly communicate with vehicle $i \in \mathcal{V}$ is denoted as $\mathcal{J}_{i, t}=\{j \in \mathcal{V}$ : $\left.\left\|\mathbf{p}_{i, t}^{(\mathrm{V})}-\mathbf{p}_{j, t}^{(\mathrm{V})}\right\| \leq R_{c}\right\}$, where $R_{c}$ is the communication range. The scenario also involves a set $\mathcal{F}=\left\{1, \ldots, N_{f}\right\}$ of $N_{f}$ noncooperative features (e.g., pedestrians, traffic lights or inactive cars) described by their position $\mathbf{p}_{k, t}^{(\mathrm{F})}=\left[p_{x_{k, t}}^{(\mathrm{F})} p_{y_{k, t}}^{(\mathrm{F})}\right]^{\mathrm{T}} \in \mathbb{R}^{2 \times 1}$ and velocity $\mathbf{v}_{k, t}^{(\mathrm{F})}=\left[v_{x_{k, t}}^{(\mathrm{F})} v_{y_{k, t}}^{(\mathrm{F})}\right]^{\mathrm{T}} \in \mathbb{R}^{2 \times 1}$ as well. The features are detected by passive ranging equipment available at vehicles. Thus, each vehicle has a measurement of the relative position-velocity of all the surrounding features that fall within the sensing range $R_{s}$. The subset of features detected by vehicle $i$ is defined as $\mathcal{F}_{i, t}=\left\{k \in \mathcal{F}:\left\|\mathbf{p}_{k, t}^{(\mathrm{F})}-\mathbf{p}_{i, t}^{(\mathrm{V})}\right\| \leq R_{s}\right\}$.

The kinematics parameters of vehicle $i \in \mathcal{V}$ are collected into the state vector $\mathbf{x}_{i, t}^{(\mathrm{V})}$ that is assumed to evolve over time $t$ according to the inertial sensor model $^{1}[52]$ :

$$
\mathbf{x}_{i, t}^{(\mathrm{V})}=\left[\begin{array}{c}
\mathbf{p}_{i, t}^{(\mathrm{V})} \\
\mathbf{v}_{i, t}^{(\mathrm{V})}
\end{array}\right]=\mathbf{A} \mathbf{x}_{i, t-1}^{(\mathrm{V})}+\mathbf{B} \mathbf{a}_{i, t-1}^{(\mathrm{V})}+\mathbf{w}_{i, t-1}^{(\mathrm{V})},
$$

\footnotetext{
${ }^{1}$ Higher order models could be used to slightly increase the localization performance [51], but they are not expected to significantly impact the analysis of the $\mathrm{V} 2 \mathrm{~V}$ cooperation gain.
}

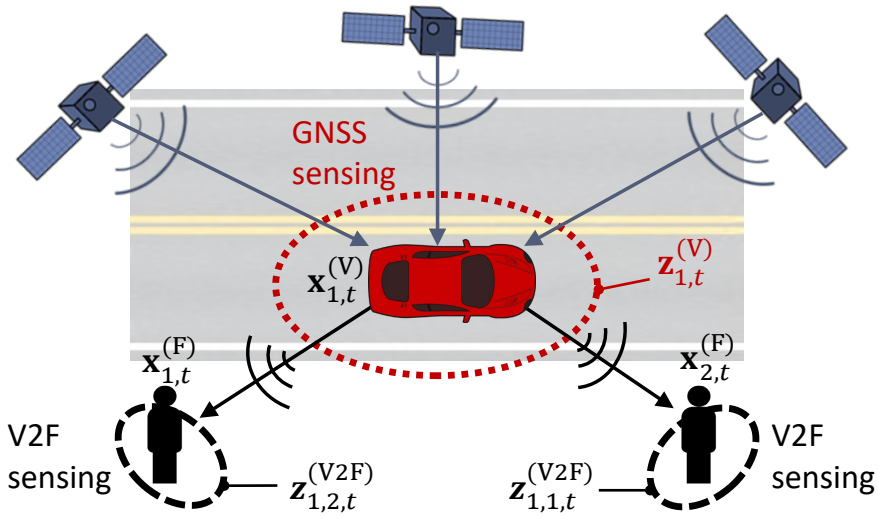

Fig. 2: Focus on ego vehicle sensors: GNSS and V2F sensing.

with

$$
\mathbf{A}=\left[\begin{array}{cc}
\mathbf{I}_{2} & T_{s} \mathbf{I}_{2} \\
\mathbf{0}_{2 \times 2} & \mathbf{I}_{2}
\end{array}\right] \quad \text { and } \quad \mathbf{B}=\left[\begin{array}{c}
0.5 T_{s}^{2} \mathbf{I}_{2} \\
T_{s} \mathbf{I}_{2}
\end{array}\right] .
$$

The matrix $\mathbf{A}$ describes the state transition while $\mathbf{B}$ relates the vehicle state to the acceleration information $\mathbf{a}_{i, t-1}^{(\mathrm{V})} \in \mathbb{R}^{2 \times 1}$, provided by an on-board inertial sensor. $T_{s}$ is the sampling interval and $\mathbf{w}_{i, t-1}^{(\mathrm{V})}$ is the Gaussian driving noise (modeling sensor measurement error and non-deterministic behaviors not accounted for by matrices $\mathbf{A}$ and $\mathbf{B}$ ) with probability density function (pdf) $p\left(\mathbf{w}_{i, t-1}^{(\mathrm{V})}\right)=\mathcal{N}\left(\mathbf{w}_{i, t-1}^{(\mathrm{V})} ; \mathbf{0}, \mathbf{Q}_{i, t-1}^{(\mathrm{V})}\right)$, where $\mathbf{Q}_{k, t-1}^{(\mathrm{V})} \in \mathbb{R}^{4 \times 4}$.

Similarly, the state $\mathbf{x}_{k, t}^{(\mathrm{F})}$ of each feature $k \in \mathcal{F}$ evolves over time $t$ according to the first order Markov model:

$$
\mathbf{x}_{k, t}^{(\mathrm{F})}=\left[\begin{array}{c}
\mathbf{p}_{k, t}^{(\mathrm{F})} \\
\mathbf{v}_{k, t}^{(\mathrm{F})}
\end{array}\right]=\mathbf{A} \mathbf{x}_{k, t-1}^{(\mathrm{F})}+\mathbf{w}_{k, t-1}^{(\mathrm{F})},
$$

where $\mathbf{w}_{k, t-1}^{(\mathrm{F})}$ is the Gaussian driving noise with pdf $p\left(\mathbf{w}_{k, t-1}^{(\mathrm{F})}\right)=\mathcal{N}\left(\mathbf{w}_{k, t-1}^{(\mathrm{F})} ; \mathbf{0}, \mathbf{Q}_{k, t-1}^{(\mathrm{F})}\right)$ and $\mathbf{Q}_{k, t-1}^{(\mathrm{F})} \in \mathbb{R}^{4 \times 4}$.

As highlighted in Fig. 2, each vehicle is assumed to gather two different types location measurements. The first one is the measurement provided by a GNSS receiver of the vehicle state (indicated with the red dotted line) which is modeled as:

$$
\boldsymbol{z}_{i, t}^{(\mathrm{V})}=\mathbf{x}_{i, t}^{(\mathrm{V})}+\mathbf{n}_{i, t}^{(\mathrm{V})},
$$

where the GNSS measurement noise $\mathbf{n}_{i, t}^{(\mathrm{V})}$ has pdf $p\left(\mathbf{n}_{i, t}^{(\mathrm{V})}\right)=$ $\mathcal{N}\left(\mathbf{n}_{i, t}^{(\mathrm{V})} ; \mathbf{0}, \mathbf{R}_{i, t}^{(\mathrm{V})}\right)$, with covariance $\mathbf{R}_{i, t}^{(\mathrm{V})} \in \mathbb{R}^{4 \times 4}$. Moreover, each vehicle uses on board ranging sensors to collect a set of V2F observation $\mathcal{O}_{i, t}=\left\{1, \ldots, O_{i, t}\right\}$ of the surrounding features $k \in \mathcal{F}_{i, t}$. Note that these are relative location/velocity measurements referred to vehicle $i$ and their association to the features is unknown. Assuming that at vehicle $i$ each feature $k \in \mathcal{F}_{i, t}$ can generate at most one $\mathrm{V} 2 \mathrm{~F}$ measurement $\ell \in \mathcal{O}_{i, t}$, the relative $\mathrm{V} 2 \mathrm{~F}$ observation is

$$
\boldsymbol{z}_{i, \ell, t}^{(\mathrm{V} 2 \mathrm{~F})}=\mathbf{x}_{k, t}^{(\mathrm{F})}-\mathbf{x}_{i, t}^{(\mathrm{V})}+\mathbf{n}_{i, \ell, t}^{(\mathrm{V} 2 \mathrm{~F})}
$$

where $k$ is the (unknown) feature associated to measurement $\ell$ and the V2F uncertainty $\mathbf{n}_{i, \ell, t}^{(\mathrm{V} 2 \mathrm{~F})}$ has pdf $p\left(\mathbf{n}_{i, \ell, t}^{(\mathrm{V} 2 \mathrm{~F})}\right)=$ $\mathcal{N}\left(\mathbf{n}_{i, \ell, t}^{(\mathrm{V} 2 \mathrm{~F})} ; \mathbf{0}, \mathbf{R}_{i, \ell, t}^{(\mathrm{V} 2 \mathrm{~F})}\right)$, with covariance $\mathbf{R}_{i, \ell, t}^{(\mathrm{V} 2 \mathrm{~F})} \in \mathbb{R}^{4 \times 4}$.

The V2F measurements available at each vehicle are shared with neighbors in order to enable a cooperative sensing of 
a)

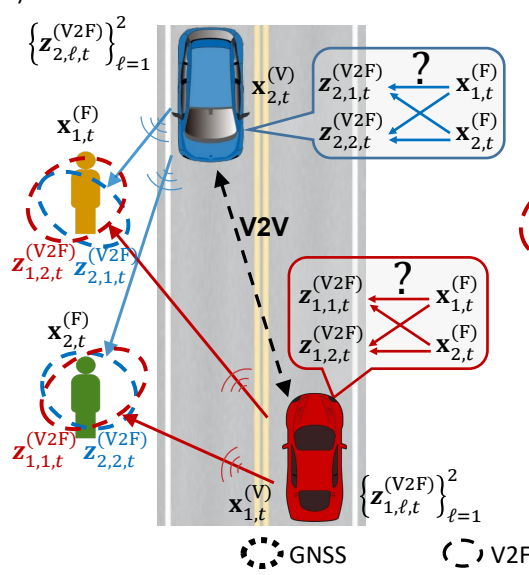

b)

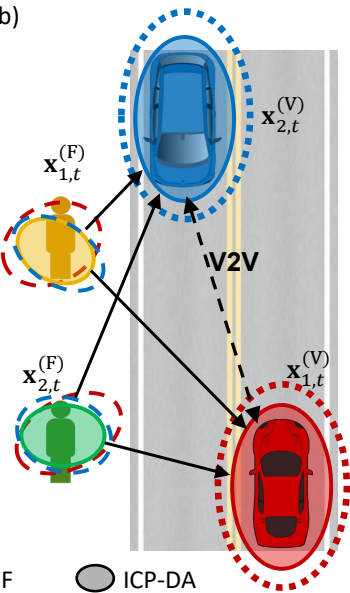

Fig. 3: a) Joint sensing of features $\mathbf{x}_{k, t}^{(\mathrm{F})}, k \in\{1,2\}$, by vehicles $\mathbf{x}_{i, t}^{(\mathrm{V})}, i \in\{1,2\}$, with unknown measurement-feature association. b) Consistent combination of measurements after the association, for feature localization (filled ellipses) and enhancement of GNSS accuracy (contours)

the surrounding environment with enhanced localization performance. This process is a part of the ICP method which is described in the following sections.

\section{DA PRoblem: The ICP-DA APPROACH}

The ICP method is a cooperative technique where the GNSS positioning of the ego vehicle is augmented by combining local GNSS data with high-resolution information on the detected features shared with connected vehicles. The vehicle initial location estimate $\boldsymbol{z}_{i, t}^{(\mathrm{V})}$ provided by the GNSS is refined by a cooperative iterative procedure that allows to fuse the $\mathrm{V} 2 \mathrm{~F}$ measurements $\boldsymbol{z}_{j, \ell, t}^{(\mathrm{V} 2 \mathrm{~F})}$ of multiple vehicles $j \in \mathcal{V}$ through the $\mathrm{V} 2 \mathrm{~V}$ links. The availability of multiple observations of a same feature at different vehicles enables in fact to localize these objects with an enhanced accuracy and, in turn, to improve positioning of the vehicles themselves. However, as features are passively detected, they do not provide any identification; when multiple observations have to be fused together, a main issue is the association between features and the relative V2F measurements.

The ICP-DA working principle is illustrated in Fig. 3, where two vehicles jointly sense two features. As shown in Fig. 3-(a), in order to consistently fuse the measurements collected by the two vehicles, each vehicles $i \in\{1,2\}$ needs at first to pair each observation $\boldsymbol{z}_{i, \ell, t}^{(\mathrm{V} 2 \mathrm{~F})}, \ell \in\{1,2\}$, with the corresponding feature $k \in\{1,2\}$ that generated that measurement. In the specific example, the observation $\ell=2$ at vehicle $i=1, \boldsymbol{z}_{1,2, t}^{(\mathrm{V} 2 \mathrm{~F})}$, originated from feature $k=1$, must be fused with the observation generated by the same feature at the second vehicle, i.e. the measurement $\boldsymbol{z}_{2,1, t}^{(\mathrm{V} 2 \mathrm{~F})}$ indexed as $\ell=1$ at vehicle $i=2$, and not with $\boldsymbol{z}_{2,2, t}^{(\mathrm{V} 2 \mathrm{~F})}$. Once the feature-observation association is solved at each vehicle, the two measurements of each feature can be combined to get a more accurate information on each feature location as shown in Fig. 3-(b) and, as a consequence, an enhanced localization of both vehicles observing the features.

For the design of the ICP-DA method, we propose to solve the association problem jointly with localization using
TABLE I: Definitions of main variables at time $t$.

\begin{tabular}{lll} 
Symbol & Dimension & Meaning \\
\hline $\mathbf{x}_{i, t}^{(\mathrm{V})}$ & $4 \times 1$ & position-velocity of veh. $i$ \\
$\mathbf{x}_{t}^{(\mathrm{V})}$ & $4 N_{v} \times 1$ & aggregated states of all vehicles \\
$\mathbf{x}_{k, t}^{(\mathrm{F})}$ & $4 \times 1$ & position-velocity of fea. $k$ \\
$\mathbf{x}_{t}^{(\mathrm{F})}$ & $4 N_{f} \times 1$ & aggregated states of all features \\
$\boldsymbol{\theta}_{t}$ & $4\left(N_{v}+N_{f}\right) \times 1$ & global vehicle and feature states \\
$\boldsymbol{z}_{i, t}^{(\mathrm{V})}$ & $4 \times 1$ & GNSS measurement at veh. $i$ \\
$\boldsymbol{z}_{t}^{(\mathrm{V})}$ & $4 N_{v} \times 1$ & aggregated GNSS measurements \\
$\boldsymbol{z}_{i, \ell, t}^{(\mathrm{V} 2 \mathrm{~F})}$ & $4 \times 1$ & V2F measurement $\ell$ at veh. $i$ \\
$\boldsymbol{z}_{i, t}^{(\mathrm{V} 2 \mathrm{~F})}$ & $4 \mathcal{O}_{i, t} \times 1$ & overall V2F measurements at veh. $i$ \\
$\boldsymbol{z}_{t}^{(\mathrm{V} 2 \mathrm{~F})}$ & $4 O \times 1$ & aggregated V2F measurements \\
$\boldsymbol{z}_{t}$ & $4\left(N_{v}+O\right) \times 1$ & global GNSS and V2F measurements \\
$\alpha_{i, k, t}$ & $1 \times 1$ & $\mathrm{~F} \rightarrow \mathrm{O}$ association for fea. $k$ at veh. $i$ \\
$\boldsymbol{\alpha}_{i, t}$ & $N_{f} \times 1$ & overall $\mathrm{F} \rightarrow \mathrm{O}$ associations at veh. $i$ \\
$\boldsymbol{\alpha}_{t}$ & $N_{v} N_{f} \times 1$ & global $\mathrm{F} \rightarrow \mathrm{O}$ associations \\
$\beta_{i, \ell, t}$ & $1 \times 1$ & O $\rightarrow \mathrm{F}$ association for meas. $\ell$ at veh. $i$ \\
$\boldsymbol{\beta}_{i, t}$ & $\mathcal{O}_{i, t} \times 1$ & overall $\mathrm{O} \rightarrow \mathrm{F}$ associations at veh. $i$ \\
$\boldsymbol{\beta}_{t}$ & $O \times 1$ & global $\mathrm{O} \rightarrow \mathrm{F}$ associations \\
\hline
\end{tabular}

a Bayesian approach. Let $\mathbf{x}_{t}^{(\mathrm{V})}=\left[\mathbf{x}_{i, t}^{(\mathrm{V})}\right]_{i \in \mathcal{V}} \in \mathbb{R}^{4 N_{v} \times 1}$ and $\mathbf{x}_{t}^{(\mathrm{F})}=\left[\mathbf{x}_{k, t}^{(\mathrm{F})}\right]_{k \in \mathcal{F}} \in \mathbb{R}^{4 N_{f} \times 1}$ be the vehicles' and features' states at time $t$, and $\boldsymbol{\theta}_{t}=\left[\mathbf{x}_{t}^{(\mathrm{V})^{\mathrm{T}}} \mathbf{x}_{t}^{(\mathrm{F})^{\mathrm{T}}}\right]^{\mathrm{T}} \in \mathbb{R}^{\left(4 N_{v}+4 N_{f}\right) \times 1}$ the combined state vector for the overall set of connected vehicles and sensed features. A complete recap of variables, with their dimensions and meanings is presented in Table I.

Considering the ideal case where a central processing unit, e.g., a road side unit (RSU), can aggregate all the GNSS measurements $\boldsymbol{z}_{t}^{(\mathrm{V})}=\left[\boldsymbol{z}_{i, t}^{(\mathrm{V})}\right]_{i \in \mathcal{V}} \in \mathbb{R}^{4 N_{v} \times 1}$ and the V2F measurements $\boldsymbol{z}_{i, t}^{(\mathrm{V} 2 \mathrm{~F})}=\left[\boldsymbol{z}_{i, \ell, t}^{(\mathrm{V} 2 \mathrm{~F})}\right]_{\ell \in \mathcal{O}_{i, t}} \in \mathbb{R}^{4 O_{i, t} \times 1}$ into a vector $\boldsymbol{z}_{t}^{(\mathrm{V} 2 \mathrm{~F})}=\left[\boldsymbol{z}_{i, t}^{(\mathrm{V} 2 \mathrm{~F})}\right]_{i \in \mathcal{V}} \in \mathbb{R}^{4 O \times 1}$, with $O=\sum_{i=1}^{N_{v}} O_{i, t}$ denoting the total number of V2F observations, the Minimum Mean Square Error (MMSE) estimate of the vehicle-feature states based on the aggregated data $\boldsymbol{z}_{t}=\left[\boldsymbol{z}_{t}^{(\mathrm{V})^{\mathrm{T}}} \boldsymbol{z}_{t}^{(\mathrm{V} 2 \mathrm{~F})^{\mathrm{T}}}\right]^{\mathrm{T}}$ can be calculated as:

$$
\hat{\boldsymbol{\theta}}_{t \mid t}=\left[\begin{array}{c}
\hat{\mathbf{x}}_{t \mid t}^{(\mathrm{V})} \\
\hat{\mathbf{x}}_{t \mid t}^{(\mathrm{F})}
\end{array}\right]=\int \boldsymbol{\theta}_{t} p\left(\boldsymbol{\theta}_{t} \mid \boldsymbol{z}_{1: t}\right) d \boldsymbol{\theta}_{t},
$$

where $\boldsymbol{z}_{1: t}=\left[\boldsymbol{z}_{\tau}\right]_{\tau=1, \ldots, t}$ collects all measurements up to time $t$ and $p\left(\boldsymbol{\theta}_{t} \mid \boldsymbol{z}_{1: t}\right)$ is the pdf of the vehicle-feature state.

The evaluation of $p\left(\boldsymbol{\theta}_{t} \mid \boldsymbol{z}_{1: t}\right)$ requires a preliminary pairing of the measurements $\boldsymbol{z}_{t}^{(\mathrm{V} 2 \mathrm{~F})}$ with the features states in $\boldsymbol{\theta}_{t}$, in order to compute the related observation likelihoods. To this aim, we model the association problem according to [44] by introducing the feature-oriented $(\mathrm{F} \rightarrow \mathrm{O})$ association variable $\alpha_{i, k, t}, \forall i \in \mathcal{V} \wedge k \in \mathcal{F}$, defined as:

$\alpha_{i, k, t}= \begin{cases}\ell \in \mathcal{O}_{i, t} & \text { if at time } t \text { feature } k \text { generates } \\ \text { measurement } \ell \text { at vehicle } i \\ 0 \begin{array}{l}\text { if at time } t \text { feature } k \text { does not generate any } \\ \left.\text { measurement at vehicle } i \text { (i.e., } k \notin \mathcal{F}_{i, t}\right) .\end{array}\end{cases}$

which relates each observation $\ell \in \mathcal{O}_{i, t}$ of vehicle $i$ to the corresponding feature $k \in \mathcal{F}_{i, t}$. Defining the $N_{f} \times 1$ stacked association vector of all the features sensed by vehicle $i$ as $\boldsymbol{\alpha}_{i, t}=\left[\alpha_{i, k, t}\right]_{k \in \mathcal{F}}$ and the overall $N_{v} N_{f} \times 1$ association vector at time $t$ as $\boldsymbol{\alpha}_{t}=\left[\boldsymbol{\alpha}_{i, t}\right]_{i \in \mathcal{V}}$, we model $\boldsymbol{\alpha}_{t}$ as a random 
process and we evaluate the posterior pdf of the vehicle-feature dynamic states as:

$$
p\left(\boldsymbol{\theta}_{t} \mid \boldsymbol{z}_{1: t}\right)=\sum_{\boldsymbol{\alpha}_{t} \in \mathcal{A}} p\left(\boldsymbol{\theta}_{t}, \boldsymbol{\alpha}_{t} \mid \boldsymbol{z}_{1: t}\right)=\sum_{\boldsymbol{\alpha}_{t} \in \mathcal{A}} p\left(\boldsymbol{\theta}_{t} \mid \boldsymbol{\alpha}_{t}, \boldsymbol{z}_{1: t}\right) p\left(\boldsymbol{\alpha}_{t} \mid \boldsymbol{z}_{1: t}\right)
$$

where the summation is over the set $\mathcal{A}$ of all the admissible association values $\boldsymbol{\alpha}_{t}$, i.e. such that each feature can generate at most one measurement at vehicle $i$ and each measurement at vehicle $i$ can be generated by at most one feature.

Since a centralized solution is unfeasible in vehicular scenarios, in the following section, we propose a distributed method for the evaluation of the marginal pdf of (7) at each vehicle. The method extends the former ICP method for known association [40], by the integration of an algorithm that solves the association problem through the computation of the distribution $p\left(\boldsymbol{\alpha}_{t} \mid \boldsymbol{z}_{1: t}\right)$. Note that, differently from [40], here the pdf (7) is not Gaussian due to the association ambiguity. Thereby, we design the ICP-DA method leveraging on a PFbased approach for joint feature-observation association and tracking of the vehicle-feature dynamics.

\section{Distributed ICP-DA Method}

To enable a decentralized fusion of the location information collected by multiple vehicles on the surrounding features, in this section we present a distributed method for implementing the ICP-DA method in Sec. III based on belief propagation. The computation of $p\left(\boldsymbol{\theta}_{t}, \boldsymbol{\alpha}_{t} \mid \boldsymbol{z}_{1: t}\right)$ in (7) is distributed over the vehicles by factorizing $p\left(\boldsymbol{\theta}_{1: t}, \boldsymbol{\alpha}_{1: t} \mid \boldsymbol{z}_{1: t}\right)$ and performing a sequential approximate marginalization of this joint pdf by BPA over a factor graph (FG) representing the factorization [53]. In the following, we first derive the factorization (Sec. IV-A) and then the distributed ICP-DA method based on BPA and consensus algorithms (Sec. IV-B). Finally, a PF-based implementation of the ICP-DA method is discussed (Sec. IV-C) together with a hard-decision low-complexity algorithm (Sec. IV-D).

\section{A. Factorization of the Joint Posterior Pdf}

Following the approach in [44], in addition to the featureoriented $(\mathrm{F} \rightarrow \mathrm{O})$ association variable $\alpha_{i, k, t}$, to model the association we introduce the observation-oriented $(\mathrm{O} \rightarrow \mathrm{F})$ association variable $\beta_{i, \ell, t}$ defined as: $\beta_{i, \ell, t}=k$, if at time $t$ the measurement $\ell$ at vehicle $i$ is generated by feature $k \in \mathcal{F}_{i, t}, \beta_{i, \ell, t}=0$, if the measurement $\ell$ is not related to any feature. ${ }^{2}$ Though this formulation is redundant - as $\beta_{i, \ell, t}$ can be derived from $\alpha_{i, k, t}$ and vice-versa - the use of $\alpha_{i, k, t}$ alongside with $\beta_{i, \ell, t}$ allows to efficiently enforce the constraint that at any time $t$ each feature $k$ can generate at most one measurement $\ell$ at vehicle $i$ and vice-versa (i.e., a one-to-one F-O mapping). This is enabled by introducing the exclusionenforcing function

$$
\Psi\left(\alpha_{i, k, t}, \beta_{i, \ell, t}\right)=\left\{\begin{aligned}
& 0, \text { if }\left(\alpha_{i, k, t}=\ell \wedge \beta_{i, \ell, t} \neq k\right) \vee \\
&\left(\alpha_{i, k, t} \neq \ell \wedge \beta_{i, \ell, t}=k\right) \\
& 1, \quad \text { otherwise }
\end{aligned}\right.
$$

\footnotetext{
${ }^{2}$ Despite the fact that clutter measurements are not present in the considered scenario, the case $\beta_{i, \ell, t}=0$ is introduced to exclude ambiguous measurements and reduce errors during the detection process as it will be detailed in the performance analysis.
}

which is null for any inconsistent $\mathrm{F}-\mathrm{O}$ pairing.

Let the $O_{i, t} \times 1$ vector $\boldsymbol{\beta}_{i, t}=\left[\boldsymbol{\beta}_{i, \ell, t}\right]_{\ell \in \mathcal{O}_{i, t}}$ and the $O \times 1$ vector $\boldsymbol{\beta}_{t}=\left[\boldsymbol{\beta}_{i, t}\right]_{i \in \mathcal{V}}$ collect all the $\mathrm{O} \rightarrow \mathrm{F}$ associations for, respectively, vehicle $i$ and all vehicles, the joint posterior pdf of the association and the vehicle-feature states $p\left(\boldsymbol{\theta}_{1: t}, \boldsymbol{\alpha}_{1: t} \mid \boldsymbol{z}_{1: t}\right)$ can be reformulated as $p\left(\boldsymbol{\theta}_{1: t}, \boldsymbol{\alpha}_{1: t}, \boldsymbol{\beta}_{1: t} \mid \boldsymbol{z}_{1: t}\right)$ and factorized as follows (see proof in appendix A):

$$
\begin{aligned}
p\left(\boldsymbol{\theta}_{1: t},\right. & \left.\boldsymbol{\alpha}_{1: t}, \boldsymbol{\beta}_{1: t} \mid \boldsymbol{z}_{1: t}\right) \propto \\
& \left(\prod_{i=1}^{N_{v}} p\left(\mathbf{x}_{i, 0}^{(\mathrm{V})}\right) \prod_{t^{\prime}=1}^{t} p\left(\mathbf{x}_{i, t^{\prime}}^{(\mathrm{V})} \mid \mathbf{x}_{i, t^{\prime}-1}^{(\mathrm{V})}\right) p\left(\boldsymbol{z}_{i, t^{\prime}}^{(\mathrm{V})} \mid \mathbf{x}_{i, t^{\prime}}^{(\mathrm{V})}\right) \times\right. \\
& \left.\prod_{k=1}^{N_{f}} p\left(\boldsymbol{z}_{i, t^{\prime}}^{(\mathrm{V} 2 \mathrm{~F})} \mid \mathbf{x}_{i, t^{\prime}}^{(\mathrm{V})}, \mathbf{x}_{k, t^{\prime}}^{(\mathrm{F})}, \alpha_{i, k, t^{\prime}}\right) \prod_{\ell=1}^{O_{i, t^{\prime}}} \Psi\left(\alpha_{i, k, t^{\prime}}, \beta_{i, \ell, t^{\prime}}\right)\right) \times \\
& \left(\prod_{k=1}^{N_{f}} p\left(\mathbf{x}_{k, 0}^{(\mathrm{F})}\right) \prod_{t^{\prime \prime}=1}^{t} p\left(\mathbf{x}_{k, t^{\prime \prime}}^{(\mathrm{F})} \mid \mathbf{x}_{k, t^{\prime \prime}-1}^{(\mathrm{F})}\right)\right),
\end{aligned}
$$

where the V2F likelihood $p\left(\boldsymbol{z}_{i, t}^{(\mathrm{V} 2 \mathrm{~F})} \mid \mathbf{x}_{i, t}^{(\mathrm{V})}, \mathbf{x}_{k, t}^{(\mathrm{F})}, \alpha_{i, k, t}\right)$ is:

$$
\begin{aligned}
p\left(\boldsymbol{z}_{i, t}^{(\mathrm{V} 2 \mathrm{~F})} \mid \mathbf{x}_{i, t}^{(\mathrm{V})}, \mathbf{x}_{k, t}^{(\mathrm{F})}, \alpha_{i, k, t}\right)= & \begin{cases}p\left(\boldsymbol{z}_{i, \ell, t}^{(\mathrm{V} 2 \mathrm{~F})} \mid \mathbf{x}_{i, t}^{(\mathrm{V})}, \mathbf{x}_{k, t}^{(\mathrm{F})}\right), & \text { if } \alpha_{i, k, t}=\ell \in \mathcal{O}_{i, t}, \\
1 & \text { if } \alpha_{i, k, t}=0 .\end{cases}
\end{aligned}
$$

$p\left(\boldsymbol{z}_{i, \ell, t}^{(\mathrm{V} 2 \mathrm{~F})} \mid \mathbf{x}_{i, t}^{(\mathrm{V})}, \mathbf{x}_{k, t}^{(\mathrm{F})}\right)$ is Gaussian with mean $\mathbf{x}_{k, t}^{(\mathrm{F})}-\mathbf{x}_{i, t}^{(\mathrm{V})}$ and covariance $\mathbf{R}_{i, \ell, t}^{(\mathrm{V} 2 \mathrm{~F})}$, while $p\left(\boldsymbol{z}_{i, t}^{(\mathrm{V} 2 \mathrm{~F})} \mid \mathbf{x}_{i, t}^{(\mathrm{V})}, \mathbf{x}_{k, t}^{(\mathrm{F})}, \alpha_{i, k, t}=0\right)$ is set to 1 to guarantee the coherency of factorization in the case of undefined V2F measurement $\boldsymbol{z}_{i, \ell=0, t}^{(\mathrm{V} 2 \mathrm{~F})}$. Furthermore, $p\left(\mathbf{x}_{i, 0}^{(\mathrm{V})}\right)$ and $p\left(\mathbf{x}_{k, 0}^{(\mathrm{F})}\right)$ denote the prior pdfs at time $t=0$, while $p\left(\boldsymbol{z}_{i, t^{\prime}}^{(\mathrm{V})} \mid \mathbf{x}_{i, t^{\prime}}^{(\mathrm{V})}\right)$ stands for the GNSS likelihood.

This factorization enables the joint association and localization problem to be solved locally at vehicles in a distributed manner, by the exchange of messages through the V2V links, as discussed in the following section.

\section{B. BPA for Data Association and Localization}

The ICP-DA problem is solved by running a loopy BP Algorithm (BPA) [44], [46] on the factor graph (FG) in Fig. 4 describing the factorization of (9) for one time instant. For visualization purposes, the FG related to the association variables is represented by a green box in Fig. 4 and it is expanded in details in Fig. 5. According to [54], in the FG the vehicle/feature states and the association variables are depicted as circles, while the factors are shown as squares. Since the FG has cycles, the BPA is iterative and consists of the cascade of two BPAs. The first one, referring to the subgraph in Fig. 5, is a BPA for data association (BPADA) that approximates through the iterations $p=1, \ldots, P$ the marginal posterior pdf of the association variable by the belief $b_{i, k, t}^{(p)}\left(\alpha_{i, k, t}\right) \approx p^{(p)}\left(\alpha_{i, k, t} \mid \boldsymbol{z}_{i, t}\right)$. This iterative algorithm is implemented following the approach in [44]. The second BPA, for vehicle/feature localization (BPA-L), uses the BPADA output to perform the ICP localization and computes over the iterations $n=1, \ldots, N$ the beliefs of all the vehicles' and features' states: $b_{i, t}^{(n)}\left(\mathbf{x}_{i, t}^{(\mathrm{V})}\right) \approx p^{(n)}\left(\mathbf{x}_{i, t}^{(\mathrm{V})} \mid \boldsymbol{z}_{1: t}\right)$ and 


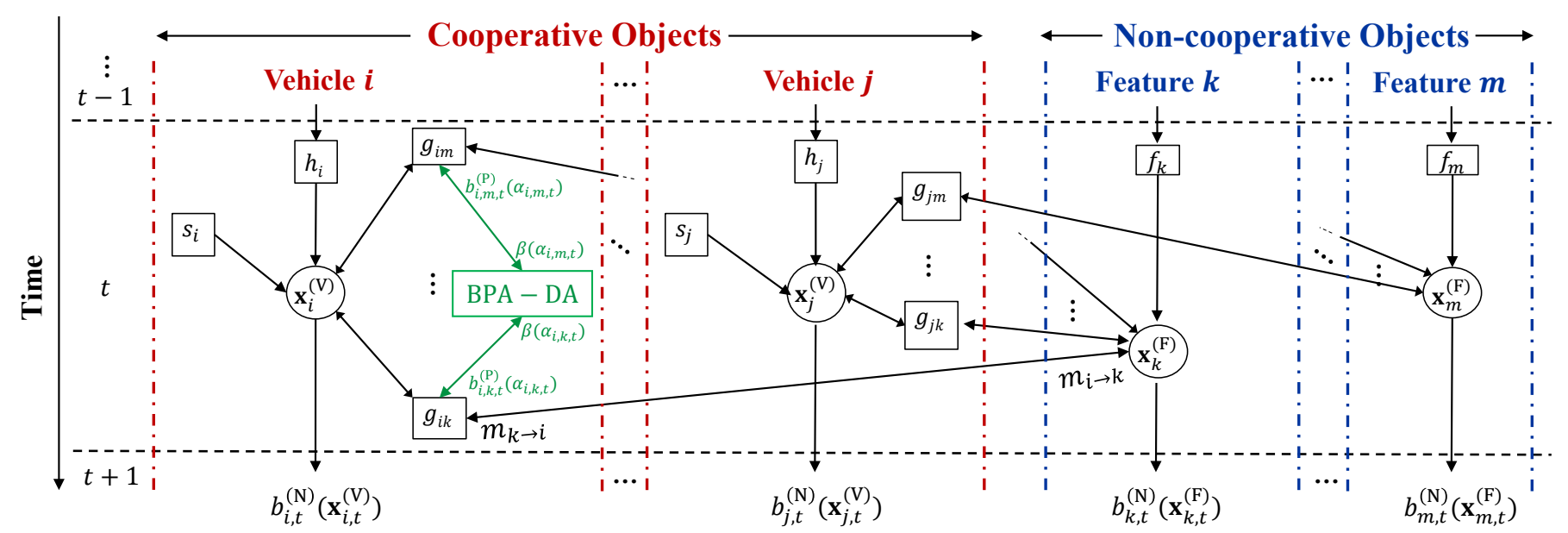

Fig. 4: Factorization of $p\left(\boldsymbol{\theta}_{t}, \boldsymbol{\alpha}_{t}, \boldsymbol{\beta}_{t} \mid \boldsymbol{z}_{1: t}\right)$ for a fixed time interval. To simplify the notation, we set $h_{i}=p\left(\mathbf{x}_{i, t}^{(\mathrm{V})} \mid \mathbf{x}_{i, t-1}^{(\mathrm{V})}\right), s_{i}=p\left(\boldsymbol{z}_{i, t}^{(\mathrm{V})} \mid \mathbf{x}_{i, t}^{(\mathrm{V})}\right), f_{k}=p\left(\mathbf{x}_{k, t}^{(\mathrm{F})} \mid \mathbf{x}_{k, t-1}^{(\mathrm{F})}\right)$, $g_{i k}=p\left(\boldsymbol{z}_{i, k, t}^{(\mathrm{V} 2 \mathrm{~F})} \mid \mathbf{x}_{i, t}^{(\mathrm{V})}, \mathbf{x}_{k, t}^{(\mathrm{F})}\right)$.

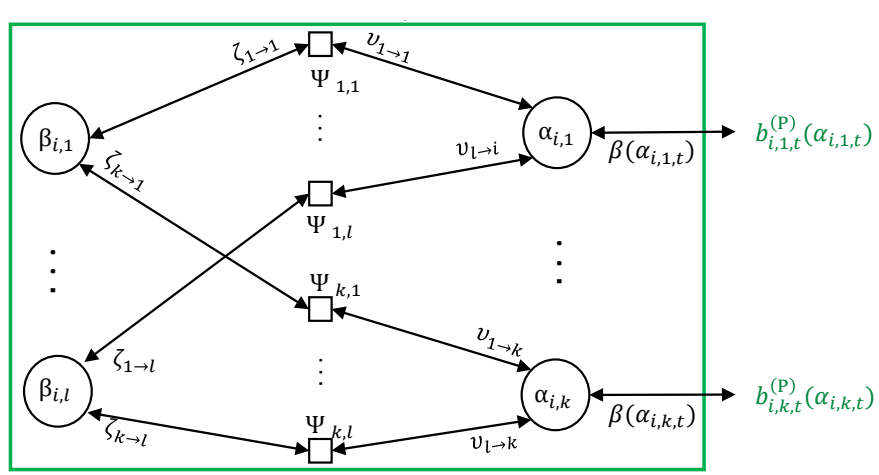

Fig. 5: Factor graph of the BPA-DA. The following notation is used: $\nu_{\ell \rightarrow k}=$ $\nu_{\ell \rightarrow k}^{(p)}\left(\alpha_{i, k, t}\right), \zeta_{k \rightarrow \ell}=\zeta_{k \rightarrow \ell}^{(p)}\left(\beta_{i, \ell, t}\right), \Psi_{k, \ell}=\Psi\left(\alpha_{i, k, t}, \beta_{i, \ell, t}\right)$

$b_{k, t}^{(n)}\left(\mathbf{x}_{k, t}^{(\mathrm{F})}\right) \approx p^{(n)}\left(\mathbf{x}_{k, t}^{(\mathrm{F})} \mid \boldsymbol{z}_{1: t}\right)$. A new solution is proposed for the BPA-L implementation based on a combination of PF, for importance sampling representation of the non-Gaussian vehicle/feature beliefs, and a log-likelihood consensus algorithm, for distributed evaluation by the vehicles of the beliefs of the states of the non-cooperative objects.

The ICP-DA algorithm consists in the following steps:

1) Prediction: The beliefs of all vehicles' and features' states at iteration $n=0$ are initialized using the beliefs of previous step, the motion prediction and the current GNSS data, as follows:

$$
\begin{aligned}
& b_{i, t}^{(0)}\left(\mathbf{x}_{i, t}^{(\mathrm{V})}\right)= \\
& p\left(\boldsymbol{z}_{i, t}^{(\mathrm{V})} \mid \mathbf{x}_{i, t}^{(\mathrm{V})}\right) \int p\left(\mathbf{x}_{i, t}^{(\mathrm{V})} \mid \mathbf{x}_{i, t-1}^{(\mathrm{V})}\right) b_{i, t-1}^{(N)}\left(\mathbf{x}_{i, t-1}^{(\mathrm{V})}\right) \mathrm{d} \mathbf{x}_{i, t-1}^{(\mathrm{V})}, \\
& b_{k, t}^{(0)}\left(\mathbf{x}_{k, t}^{(\mathrm{F})}\right)=\int p\left(\mathbf{x}_{k, t}^{(\mathrm{F})} \mid \mathbf{x}_{k, t-1}^{(\mathrm{F})}\right) b_{k, t-1}^{(N)}\left(\mathbf{x}_{k, t-1}^{(\mathrm{F})}\right) d \mathbf{x}_{k, t-1}^{(\mathrm{F})},
\end{aligned}
$$

where $b_{i, t-1}^{(N)}\left(\mathbf{x}_{i, t-1}^{(\mathrm{V})}\right)$ and $b_{k, t-1}^{(N)}\left(\mathbf{x}_{k, t-1}^{(\mathrm{F})}\right)$ are respectively the vehicle and feature beliefs at time $t-1$, $p\left(\mathbf{x}_{i, t}^{(\mathrm{V})} \mid \mathbf{x}_{i, t-1}^{(\mathrm{V})}\right)=\mathcal{N}\left(\mathbf{x}_{i, t}^{(\mathrm{V})} ; \mathbf{x}_{i, t-1}^{(\mathrm{V})}+\mathbf{B} \mathbf{a}_{i, t-1}^{(\mathrm{V})}, \mathbf{Q}_{i, t-1}^{(\mathrm{V})}\right)$ and $p\left(\mathbf{x}_{k, t}^{(\mathrm{F})} \mid \mathbf{x}_{k, t-1}^{(\mathrm{F})}\right)=\mathcal{N}\left(\mathbf{x}_{k, t}^{(\mathrm{F})} ; \mathbf{x}_{k, t-1}^{(\mathrm{F})}, \mathbf{Q}_{k, t-1}^{(\mathrm{F})}\right)$ are the transition pdfs from (1) and (2), and $p\left(\boldsymbol{z}_{i, t}^{(\mathrm{V})} \mid \mathbf{x}_{i, t}^{(\mathrm{V})}\right)=$
$\mathcal{N}\left(\boldsymbol{z}_{i, t}^{(\mathrm{V})} ; \mathbf{x}_{i, t}^{(\mathrm{V})}, \mathbf{R}_{i, t}^{(\mathrm{V})}\right)$ is the likelihood of the GNSS measurement at vehicle $i$ from (3).

2) V2F measurement evaluation for association: Vehicle $i$ evaluates each $\mathrm{O} \rightarrow \mathrm{F}$ association by integrating the $\mathrm{V} 2 \mathrm{~F}$ measurement likelihood over all possible feature locations, and weighting with the prior beliefs (11)-(12):

$\eta\left(\alpha_{i, k, t}\right)=p\left(\boldsymbol{z}_{i, t}^{(\mathrm{V} 2 \mathrm{~F})} \mid \alpha_{i, k, t}\right)=$

$\iint p\left(\boldsymbol{z}_{i, t}^{(\mathrm{V} 2 \mathrm{~F})} \mid \mathbf{x}_{i, t}^{(\mathrm{V})}, \mathbf{x}_{k, t}^{(\mathrm{F})}, \alpha_{i, k, t}\right) b_{i, t}^{(0)}\left(\mathbf{x}_{i, t}^{(\mathrm{V})}\right) b_{k, t}^{(0)}\left(\mathbf{x}_{k, t}^{(\mathrm{F})}\right) d \mathbf{x}_{i, t}^{(\mathrm{V})} d \mathbf{x}_{k, t}^{(\mathrm{F})}$.

where $\quad p\left(\boldsymbol{z}_{i, t}^{(\mathrm{V} 2 \mathrm{~F})} \mid \mathbf{x}_{i, t}^{(\mathrm{V})}, \mathbf{x}_{k, t}^{(\mathrm{F})}, \alpha_{i, k, t}\right)$ $\mathcal{N}\left(\boldsymbol{z}_{i, \alpha_{i, k, t}, t}^{(\mathrm{V} 2 \mathrm{~F})} ; \mathbf{x}_{k, t}^{(\mathrm{F})}-\mathbf{x}_{i, t}^{(\mathrm{V})}, \mathbf{R}_{i, \alpha_{i, k, t}, t}^{(\mathrm{V} 2 \mathrm{~F})}\right)$, see (10).

3) BPA-DA for measurement-feature association: Following [44], here each vehicle $i$ computes the association beliefs $b_{i, k, t}^{(\mathrm{p})}\left(\alpha_{i, k, t}\right)$ by a repeated exchange of messages from the $\mathrm{F} \rightarrow \mathrm{O}$ association variable $\alpha_{i, k, t}$ to the $\mathrm{O} \rightarrow \mathrm{F}$ association variable $\beta_{i, \ell, t}$ and vice-versa, so as to exclude inconsistent pairings (e.g., the same measurement associated to two different features) and restrict the belief evaluation to one-to-one associations. The message from $\alpha_{i, k, t}$ to $\beta_{i, \ell, t}$ is initialized at BPA-DA iteration $p=0$ as:

$$
m_{k \rightarrow \ell}^{(0)}\left(\beta_{i, \ell, t}\right)=\sum_{\alpha_{i, k, t}=0}^{O_{i, t}} \eta\left(\alpha_{i, k, t}\right) \Psi\left(\alpha_{i, k, t}, \beta_{i, \ell, t}\right),
$$

while at subsequent iterations, $p=1,2, \ldots$, the messages are updated as follows:

$$
m_{\ell \rightarrow k}^{(p)}\left(\alpha_{i, k, t}\right)=\sum_{\beta_{i, \ell, t}=1}^{\left|\mathcal{F}_{i, t}\right|} \Psi\left(\alpha_{i, k, t}, \beta_{i, \ell, t}\right) \prod_{k^{\prime} \in \mathcal{F} \backslash\{k\}} m_{k^{\prime} \rightarrow \ell}^{(p-1)}\left(\beta_{i, \ell, t}\right),
$$

$$
\begin{gathered}
m_{k \rightarrow \ell}^{(p)}\left(\beta_{i, \ell, t}\right)=\sum_{\alpha_{i, k, t}=0}^{O_{i, t}} \eta\left(\alpha_{i, k, t}\right) \Psi\left(\alpha_{i, k, t}, \beta_{i, \ell, t}\right) \times \\
\prod_{\ell^{\prime} \rightarrow k}^{(p)}\left(\alpha_{i, k, t}\right) .
\end{gathered}
$$


At the last iteration, $p=P$, the belief of the association variable $\alpha_{i, k, t}$ is obtained as:

$$
b_{i, k, t}^{(\mathrm{P})}\left(\alpha_{i, k, t}\right)=\prod_{\ell \in \mathcal{O}_{i, t}} m_{\ell \rightarrow k}^{(P)}\left(\alpha_{i, k, t}\right) .
$$

An efficient implementation of the above BPA-DA steps can be found in [44].

4) V2F measurement evaluation for localization: The association beliefs (17) given by BPA-DA are here used by each vehicle to compute the V2F location likelihood as:

$$
\begin{aligned}
p\left(\boldsymbol{z}_{i, t}^{(\mathrm{V} 2 \mathrm{~F})} \mid \mathbf{x}_{i, t}^{(\mathrm{V})}, \mathbf{x}_{k, t}^{(\mathrm{F})}\right)= & \\
& \sum_{\alpha_{i, k, t}=0}^{O_{i, t}} p\left(\boldsymbol{z}_{i, t}^{(\mathrm{V} 2 \mathrm{~F})} \mid \mathbf{x}_{i, t}^{(\mathrm{V})}, \mathbf{x}_{k, t}^{(\mathrm{F})}, \alpha_{i, k, t}\right) b_{i, k, t}^{(\mathrm{P})}\left(\alpha_{i, k, t}\right),
\end{aligned}
$$

for the ICP-based localization in the next step.

5) BPA-L for localization: The BPA algorithm for vehicle/feature localization approximates the posterior pdf of the vehicles' and features' states over the iterations $n$ by the beliefs $b_{i, t}^{(n)}\left(\mathbf{x}_{i, t}^{(\mathrm{V})}\right), \forall i$, and $b_{k, t}^{(n)}\left(\mathbf{x}_{k, t}^{(\mathrm{F})}\right), \forall k$. The procedure starts from the vehicle/feature beliefs obtained at step $n-1$. In order to evaluate a belief for the $k$ th feature state, $\mathbf{x}_{k, t}^{(\mathrm{F})}$, vehicle $i$ combines the belief of its own state $\mathbf{x}_{i, t}^{(\mathrm{V})}$ from step $n-1$ with the V2F likelihood $p\left(\boldsymbol{z}_{i, t}^{(\mathrm{V} 2 \mathrm{~F})} \mid \mathbf{x}_{i, t}^{(\mathrm{V})}, \mathbf{x}_{k, t}^{(\mathrm{F})}\right)$ based on (18), providing the following message from vehicle $i$ to feature $k$ :

$m_{i \rightarrow k}^{(n)}\left(\mathbf{x}_{k, t}^{(\mathrm{F})}\right) \propto \int \frac{b_{i, t}^{(n-1)}\left(\mathbf{x}_{i, t}^{(\mathrm{V})}\right)}{m_{k \rightarrow i}^{(n-1)}\left(\mathbf{x}_{i, t}^{(\mathrm{V})}\right)} p\left(\boldsymbol{z}_{i, t}^{(\mathrm{V} 2 \mathrm{~F})} \mid \mathbf{x}_{i, t}^{(\mathrm{V})}, \mathbf{x}_{k, t}^{(\mathrm{F})}\right) d \mathbf{x}_{i, t}^{(\mathrm{V})}$

All the messages received by feature $k$ are then gathered to compute the refined belief as follows:

$$
b_{k, t}^{(n)}\left(\mathbf{x}_{k, t}^{(\mathrm{F})}\right) \propto b_{k, t}^{(0)}\left(\mathbf{x}_{k, t}^{(\mathrm{F})}\right) \prod_{i \in \mathcal{V}_{k, t}} m_{i \rightarrow k}^{(n)}\left(\mathbf{x}_{k, t}^{(\mathrm{F})}\right),
$$

where $\mathcal{V}_{k, t}$ is the set of vehicles that sense feature $k$. Note that if a feature $\mathbf{x}_{k, t}^{(\mathrm{F})}$ is not observed by any vehicle $i \in \mathcal{V}$, the related belief (20) is reset to a uniform distribution. Based on the updated feature's belief (20) and the V2F likelihood, vehicle $i$ receives the following message from feature $k$ :

$$
m_{k \rightarrow i}^{(n)}\left(\mathbf{x}_{i, t}^{(\mathrm{V})}\right) \propto \int \frac{b_{k, t}^{(n)}\left(\mathbf{x}_{k, t}^{(\mathrm{F})}\right)}{m_{i \rightarrow k}^{(n)}\left(\mathbf{x}_{k, t}^{(\mathrm{F})}\right)} p\left(\boldsymbol{z}_{i, t}^{(\mathrm{V} 2 \mathrm{~F})} \mid \mathbf{x}_{i, t}^{(\mathrm{V})}, \mathbf{x}_{k, t}^{(\mathrm{F})}\right) d \mathbf{x}_{k, t}^{(\mathrm{F})} .
$$

Finally, vehicle $i$ refines its own belief by combining the local information with all messages coming from the sensed features $k \in \mathcal{F}_{i, t}$ :

$$
b_{i, t}^{(n)}\left(\mathbf{x}_{i, t}^{(\mathrm{V})}\right) \propto b_{i, t}^{(0)}\left(\mathbf{x}_{i, t}^{(\mathrm{V})}\right) \prod_{k \in \mathcal{F}_{i, t}} m_{k \rightarrow i}^{(n)}\left(\mathbf{x}_{i, t}^{(\mathrm{V})}\right) .
$$

The BPA-L steps (19)-(22) are repeated until convergence is reached.

In the above algorithm we treated the features as cooperative entities that are actively involved in the message passing procedure. However, being passive objects, features cannot compute their own belief in (20) nor communicate with vehicles. Following [40], this problem can be solved by using a consensus algorithm which enables vehicles to compute the feature belief in (20) in a distributed way by exchanging data through V2V links. More details are given in the following section where a PF-based implementation is discussed.

\section{ICP-DA PF-based Implementation}

The introduction of the non-Gaussian variables, $\alpha_{i, k, t}$ and $\beta_{i, \ell, t}$, makes the use of Kalman filtering, as originally proposed in [40], suboptimal for the extension of ICP with data association. To deal with non-Gaussian statistics, we propose a PF-based ICP-DA algorithm (ICP-DA-PF) where each vehicle belief $b_{i, t}^{(n)}\left(\mathbf{x}_{i, t}^{(\mathrm{V})}\right)$ is described by the set of $J_{\mathrm{v}}$ particles and related weights, $\left\{\mathbf{s}_{i, t}^{(v)}, w_{i, t}^{(v)}\right\}_{v=1}^{J_{\mathrm{v}}}, \forall i \in \mathcal{V}$, and similarly each feature belief $b_{k, t}^{(n)}\left(\mathbf{x}_{k, t}^{(\mathrm{F})}\right)$ is represented by the set of $J_{\mathrm{f}}$ particles with associated weights $\left\{\mathbf{s}_{k, t}^{(f)}, w_{k, t}^{(f)}\right\}_{f=1}^{J_{\mathrm{f}}}, \forall k \in \mathcal{F}$. The iteration index $n$ is dropped to simplify the notation. The computation of particles and weights is performed as described below.

Following the BPA steps in previous section, the vehicle and feature beliefs (20)-(22) are initialized for step 1 as:

$$
\begin{aligned}
& b_{i, t}^{(0)}\left(\mathbf{x}_{i, t}^{(\mathrm{V})}\right) \approx \frac{1}{J_{\mathrm{v}}} \sum_{v=1}^{J_{\mathrm{v}}} p\left(\boldsymbol{z}_{i, t}^{(\mathrm{V})} \mid \mathbf{s}_{i, t}^{(v)}\right) \delta\left(\mathbf{x}_{i, t}^{(\mathrm{V})}-\mathbf{s}_{i, t}^{(v)}\right), \\
& b_{k, t}^{(0)}\left(\mathbf{x}_{k, t}^{(\mathrm{F})}\right) \approx \frac{1}{J_{\mathrm{f}}} \sum_{f=1}^{J_{\mathrm{f}}} \delta\left(\mathbf{x}_{k, t}^{(\mathrm{F})}-\mathbf{s}_{k, t}^{(f)}\right),
\end{aligned}
$$

with equal weights and particles obtained from the propagation of the particles associated with the previous timeinstant beliefs according to the dynamic models (1)-(2). The GNSS likelihood function is evaluated for each particle as $p\left(\boldsymbol{z}_{i, t}^{(\mathrm{V})} \mid \mathbf{s}_{i, t}^{(v)}\right)=\mathcal{N}\left(\boldsymbol{z}_{i, t}^{(\mathrm{V})} ; \mathbf{s}_{i, t}^{(v)}, \mathbf{R}_{i, t}^{(\mathrm{V})}\right)$ according to (3), while $\delta(\cdot)$ denotes the multidimensional Dirac delta function.

In step 2, the likelihood function for association can be computed by inserting (23)-(24) in (13) as follows:

$$
\begin{aligned}
\eta\left(\alpha_{i, k, t}\right) & \approx \\
& \frac{1}{J_{\mathrm{v}} J_{\mathrm{f}}} \sum_{v=1}^{J_{\mathrm{v}}} \sum_{f=1}^{J_{\mathrm{f}}} p\left(\boldsymbol{z}_{i, t}^{(\mathrm{V})} \mid \mathbf{s}_{i, t}^{(v)}\right) p\left(\boldsymbol{z}_{i, t}^{(\mathrm{V} 2 \mathrm{~F})} \mid \mathbf{s}_{i, t}^{(v)}, \mathbf{s}_{k, t}^{(f)}, \alpha_{i, k, t}\right),
\end{aligned}
$$

where $p\left(\boldsymbol{z}_{i, t}^{(\mathrm{V} 2 \mathrm{~F})} \mid \mathbf{s}_{i, t}^{(v)}, \mathbf{s}_{k, t}^{(f)}, \alpha_{i, k, t}\right)=\mathcal{N}\left(\boldsymbol{z}_{i, \alpha_{i, k, t}, t}^{(\mathrm{V} 2 \mathrm{~F})} ; \mathbf{s}_{k, t}^{(f)}-\right.$ $\mathbf{s}_{i, t}^{(v)}, \mathbf{R}_{i, \alpha_{i, k, t}, t}^{(\mathrm{V} 2 \mathrm{~F})}, \forall \alpha_{i, k, t} \in \mathcal{O}_{i, t}$, according to (4) and (10). The BPA-DA procedure in step 3 does not involve particles and can thus be implemented by simply using (25) in the association equations (14)-(16).

The likelihood function for localization can now be computed (as step 4) using (18) for each pair of feature-vehicle particles. The BPA-L procedure follows in step 5, where the message $m_{i \rightarrow k, t}\left(\mathbf{x}_{k, t}^{(\mathrm{F})}\right)$ as defined in (19) is represented by the set of particles and weights $\left\{\mathbf{s}_{k, t}^{(f)}, w_{i \rightarrow k, t}^{(f)}\right\}_{f=1}^{J_{\mathrm{f}}}$, with

$$
w_{i \rightarrow k, t}^{(f)} \propto \frac{1}{J_{\mathrm{v}}} \sum_{v=1}^{J_{\mathrm{v}}} p\left(\boldsymbol{z}_{i, t}^{(\mathrm{V})} \mid \mathbf{s}_{i, t}^{(v)}\right) \prod_{\substack{h \in \mathcal{F}_{i, t} \\ h \neq k}} w_{h \rightarrow i, t}^{(v)} p\left(\boldsymbol{z}_{i, t}^{(\mathrm{V} 2 \mathrm{~F})} \mid \mathbf{s}_{i, t}^{(v)}, \mathbf{s}_{h, t}^{(f)}\right) .
$$


TABLE II: BPA-DA computational complexity at each vehicle per time step.

\begin{tabular}{cccc} 
& ICP $($ known DA $)$ & ICP-DA-PF & ICP-DA-LC \\
\hline BPA-DA & - & $\mathcal{O}\left(J_{\mathrm{v}} J_{\mathrm{f}} N_{f}^{2}\right)$ & $\mathcal{O}\left(N_{f}^{2}\right)$ \\
BPA-L & $\mathcal{O}\left(N_{B P A-L} N_{\text {cons }} N_{v} N_{f}\right)$ & $\mathcal{O}\left(N_{B P A-L} N_{\text {cons }} N_{v} J_{\mathrm{v}} J_{\mathrm{f}} N_{f}\right)$ & $\mathcal{O}\left(N_{B P A-L} N_{\text {cons } s} N_{v} N_{f}\right)$ \\
\hline
\end{tabular}

The PF representation of the $k$ th feature belief in (20) is:

$$
b_{k, t}^{(n)}\left(\mathbf{x}_{k, t}^{(\mathrm{F})}\right) \propto \sum_{\mathrm{f}=1}^{J_{\mathrm{f}}} \underbrace{\frac{1}{J_{\mathrm{f}}} \prod_{i \in \mathcal{V}_{k, t}} w_{i \rightarrow k, t}^{(f)}}_{\tilde{w}_{k, t}^{(\mathrm{f})}} \delta\left(\mathbf{x}_{k, t}^{(\mathrm{F})}-\mathbf{s}_{k, t}^{(f)}\right)
$$

with $\tilde{w}_{k, t}^{(\mathrm{f})}$ denoting the weight related to feature particle $f$, that has to be normalized as $w_{k, t}^{(f)}=\frac{\tilde{w}_{k, t}^{(f)}}{\sum_{\mathrm{f}=1}^{J_{\mathrm{f}}} \tilde{w}_{k, t}^{(f)}}$. Similarly, the message from feature $k$ to vehicle $i, m_{k \rightarrow i}^{(n)}\left(\mathbf{x}_{i, t}^{(\mathrm{V})}\right)$, is represented by the set $\left\{\mathbf{s}_{i, t}^{(v)}, w_{k \rightarrow i, t}^{(v)}\right\}_{v=1}^{J_{\mathrm{v}}}$, with weights computed according to (21) as

$$
w_{k \rightarrow i, t}^{(v)} \propto \frac{1}{J_{\mathrm{f}}} \sum_{f=1}^{J_{\mathrm{f}}} \prod_{\substack{j \in \mathcal{V}_{k, t} \\ j \neq i}} w_{j \rightarrow k, t}^{(f)} p\left(\boldsymbol{z}_{i, t}^{(\mathrm{V} 2 \mathrm{~F})} \mid \mathbf{s}_{j, t}^{(v)}, \mathbf{s}_{k, t}^{(f)}\right) .
$$

The PF representation of the $i$ th vehicle belief is then given by:

$$
b_{i, t}^{(n)}\left(\mathbf{x}_{i, t}^{(\mathrm{V})}\right) \propto \sum_{v=1}^{J_{\mathrm{v}}} \underbrace{\frac{1}{J_{\mathrm{v}}} p\left(\boldsymbol{z}_{i, t}^{(\mathrm{V})} \mid \mathbf{s}_{i, t}^{(v)}\right) \prod_{k \in \mathcal{F}_{i, t}} w_{k \rightarrow i, t}^{(v)}}_{\tilde{w}_{i, t}^{(v)}} \delta\left(\mathbf{x}_{i, t}^{(\mathrm{V})}-\mathbf{s}_{i, t}^{(v)}\right),
$$

where $\tilde{w}_{i, t}^{(v)}$ is the non-normalized weight, related to vehicle particle $\mathbf{s}_{i, t}^{(\mathrm{v})}$, to be normalized as $w_{i, t}^{(v)}=\frac{\tilde{w}_{i, t}^{(v)}}{\sum_{v=1}^{J_{\mathrm{v}}} \tilde{w}_{i, t}^{(v)}}$. All vehicle and feature particles finally undergo importance resampling so as to avoid the particle degeneracy [55]. Note that identical sets of particles are assumed to be sampled at all vehicles for feature $k$ and at all features for vehicle $i$ to enable message fusion as in in (26)-(27), i.e. the set $\left\{\mathbf{s}_{k, t}^{(f)}\right\}_{f=1}^{J_{\mathrm{f}}}$ is the same for all vehicles $i \in \mathcal{V}$ and $\left\{\mathbf{s}_{i, t}^{(v)}\right\}_{v=1}^{J_{\mathrm{v}}}$ is the same for all features $k \in \mathcal{F}$. This requires local random number generators to be synchronized at all vehicles. At convergence, the estimates of the feature/vehicle states are obtained according to the MMSE criterion, respectively as $\hat{\mathbf{x}}_{k, t}^{(\mathrm{F})}=\sum_{f=1}^{J_{\mathrm{f}}} w_{k, t}^{(f)} \mathbf{s}_{k, t}^{(f)}, \forall k$, and $\hat{\mathbf{x}}_{i, t}^{(\mathrm{V})}=\sum_{v=1}^{J_{\mathrm{v}}} w_{i, t}^{(v)} \mathbf{s}_{i, t}^{(v)}, \forall i$.

The ICP-DA-PF method requires the computation of the belief (26) at each feature $k$, which is however unfeasible since features are not actively involved in the localization process. We thus propose this belief to be evaluated cooperatively by the vehicles by a consensus-based algorithm that enables the distributed computation of the features' weights $w_{k, t}^{(f)}, \forall f \in$ $J_{\mathrm{f}}, \forall k \in \mathcal{F}$. In particular, defining the product of messages over the vehicles sensing the same feature $k$ in (22) as

$$
q_{k, t}^{(f)}=\prod_{i \in \mathcal{V}_{k, t}} w_{i \rightarrow k, t}^{(f)} \forall f=1, \ldots, J_{\mathrm{f}}
$$

and taking the $\operatorname{logarithm} L\left(q_{k, t}^{(f)}\right)=\log \left(q_{k, t}^{(f)}\right)$, we get:

$$
L\left(q_{k, t}^{(f)}\right)=\sum_{i \in \mathcal{V}_{k, t}} \log \left(w_{i \rightarrow k, t}^{(f)}\right)=\frac{1}{\left|\mathcal{V}_{k, t}\right|} \sum_{i=1}^{\left|\mathcal{V}_{k, t}\right|} \log \left(w_{i \rightarrow k, t}^{(f)}\right),
$$

which is an arithmetic average that can be evaluated in a distributed manner by vehicles through average consensus [47]. We thus propose the distributed computation of the feature weights by successive refinements of local weights at vehicles based on information exchange with neighbors. Once a consensus is reached by all vehicles on the $L\left(q_{k, t}^{(\mathrm{f})}\right)$, the weight (26) is obtained as $q_{k, t}^{(\mathrm{f})}=\exp \left(L\left(q_{k, t}^{(\mathrm{f})}\right)\right)$ and is used for the evaluation of the feature belief (26). Since for finite number of iterations the consensus provides an approximation of (29) at each vehicle, a max-consensus algorithm is performed after the average consensus to obtain the same feature weights at each vehicle.

\section{ICP-DA Low-complexity Implementation}

The PF-based method presented in the previous section provides an optimal approach for solving the non-Gaussian ICPDA estimation problem. However, as the BPA-DA quadratically scales with the number of features [43], the computational complexity at single vehicle $i$ for each BPA-DA iteration scales as $\mathcal{O}\left(J_{\mathrm{v}} J_{\mathrm{f}} N_{f}^{2}\right)$. Note that the evaluation of the complexity is done considering all-to-all $\mathrm{V} 2 \mathrm{~V}$ and $\mathrm{V} 2 \mathrm{~F}$ connectivity (i.e., $\left|\mathcal{O}_{i, t}\right|=N_{f},\left|\mathcal{J}_{i, t}\right|=N_{v}$ ) and assuming $J_{\mathrm{v}} \gg N_{v}$ and $J_{\mathrm{f}} \gg N_{f}$.

To reduce the computational burden, in this section we propose a suboptimal low-complexity (LC) implementation of the ICP-DA method (ICP-DA-LC), in which a hard decision is made on the association variable at the end of BPA-DA, so as to approximate the features' beliefs with Gaussian pdfs and implement the BPA-L by a distributed Kalman filter [40] where only the first two moments of the vehicle/feature beliefs are computed. In the proposed method, each vehicle performs a Bayesian detection to decide if the feature $k \in \mathcal{F}_{i, t}$ has been sensed by its sensors, by comparing the belief of the association variable to any of its measurements with a threshold $\eta_{\mathrm{TH}}$. If $b_{i, k, t}^{(\mathrm{P})}\left(\alpha_{i, k, t}=0\right)<\eta_{\mathrm{TH}}$, the feature $k$ is detected and paired with the most probable measurement:

$$
\hat{\alpha}_{i, k, t}=\underset{\ell \in \mathcal{O}_{i, t}}{\operatorname{argmax}} b_{i, k, t}^{(\mathrm{P})}\left(\alpha_{i, k, t}\right) .
$$

With the above Maximum-A-Posteriori (MAP) decision the V2F likelihood in (18) becomes:

$$
p\left(\boldsymbol{z}_{i, t}^{(\mathrm{V} 2 \mathrm{~F})} \mid \mathbf{x}_{i, t}^{(\mathrm{V})}, \mathbf{x}_{k, t}^{(\mathrm{F})}\right)=p\left(\boldsymbol{z}_{i, t}^{(\mathrm{V} 2 \mathrm{~F})} \mid \mathbf{x}_{i, t}^{(\mathrm{V})}, \mathbf{x}_{k, t}^{(\mathrm{F})}, \hat{\alpha}_{i, k, t}\right) .
$$

The ICP-DA-LC solution still has a computational complexity that quadratically scales with the number of features, but it avoids the intensive computations of the huge number 
of particles that is required in dynamic vehicular scenarios to sample both feature and vehicle beliefs. A comparison of computational complexity of all the considered methods, namely ICP with perfect DA, ICP-DA-PF and ICP-DA-LC, evaluated at single vehicle is summarized in Table II, with $N_{\text {cons }}$ denoting the number of consensus iterations. As it can be deduced from Table II, the dominant term in the ICPDA-PF complexity is represented by the number of particles used to sample the vehicle/feature beliefs. To provide high localization accuracy, $J_{\mathrm{v}}$ and $J_{\mathrm{f}}$ have to be large enough. Note also that, while in the ICP-DA-PF implementation both the average consensus and the max-consensus algorithms are computed for each particle of the feature belief, by considering the ICP-DA-LC solution only the average consensus on the first two moments of $b_{k, t}^{(n)}\left(\mathbf{x}_{k, t}^{(\mathrm{F})}\right)$ is required [40]. Based on this, the ICP-DA-LC can provide high benefits in term of computational complexity and communication overhead.

\section{Performance Assessment in Simplified Road SCENARIOS}

In this section, we analyze the performance of the proposed cooperative positioning method in simulated road scenarios, to highlight the benefits provided by the ICP-DA approach compared to GNSS tracking solutions. We consider two scenarios, first over a single road (Sec. V-A) and then in a crossroad (Sec. V-B). The first scenario is used to compare the two different implementations of the ICP-DA method, i.e. $\mathrm{PF}$ and LC, in a controlled environment with two features, to highlight the impact of feature spacing on the association process. In the second scenario, we extend the analysis to a more complex environment with more vehicle interactions, for varying number of features and degree of feature mobility. The analysis aims at showing the improvement on vehicle localization with respect to a conventional GNSS solution. The GNSS benchmark is implemented as a non-cooperative tracking filter that uses model (1) for integrating the positionvelocity estimate provided by the GNSS receiver with the inertial sensor measurement at the single vehicle, without fusion of data from nearby vehicles. The performance assessment is also targeted to evaluate the impact of DA uncertainty and the feature mobility on the vehicle localization accuracy.

\section{A. Single-Road Scenario with Static Features}

We consider the single-road scenario in Fig. 6 with two interconnected vehicles traveling in the same direction at an average speed of $25 \mathrm{~km} / \mathrm{h}$, with space headway $5 \mathrm{~m}$. The vehicle motion is simulated according to the inertial sensor model in (1) with zero mean acceleration $\mathbf{a}_{i, t-1}^{(\mathrm{V})}$ and sampling time $T_{s}=1 \mathrm{~s}$. The covariance of the Gaussian driving noise in (1) is $\mathbf{Q}_{i, t-1}^{(\mathrm{V})}=\mathbf{B} \operatorname{diag}\left(\sigma_{a_{i}, x}^{(\mathrm{V})^{2}}, \sigma_{a_{i}, y}^{(\mathrm{V})^{2}}\right) \mathbf{B}^{\mathrm{T}}$, where $\sigma_{a_{i}, x}^{(\mathrm{V})}$ and $\sigma_{a_{i}, y}^{(\mathrm{V})}$ represent the acceleration fluctuations along the vehicle longitudinal and transversal directions. They are set to $\sigma_{a_{i}, x}^{(\mathrm{V})}=0.2 \mathrm{~m} / \mathrm{s}^{2} \sigma_{a_{i}, y}^{(\mathrm{V})}=0.001 \mathrm{~m} / \mathrm{s}^{2}$, respectively. The GNSS accuracy on the whole area is set to $\mathbf{R}_{i, t}^{(\mathrm{V})}=\operatorname{blockdiag}\left(\sigma_{p}^{(\mathrm{V})^{2}} \mathbf{I}_{2}, \sigma_{v}^{(\mathrm{V})^{2}} \mathbf{I}_{2}\right)$ with standard deviations of $\sigma_{p}^{(\mathrm{V})}=8 \mathrm{~m}$ and $\sigma_{v}^{(\mathrm{V})}=0.1 \mathrm{~m} / \mathrm{s}$, respectively. The vehicles

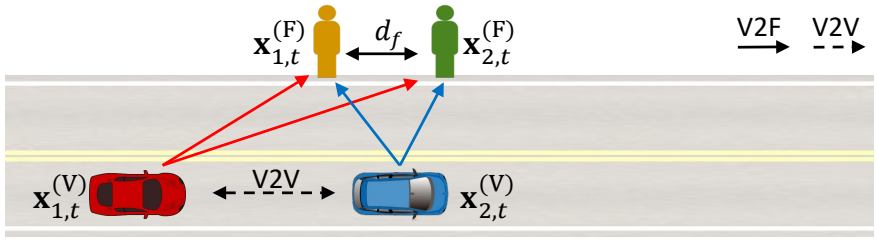

Fig. 6: Simulated single road scenario.
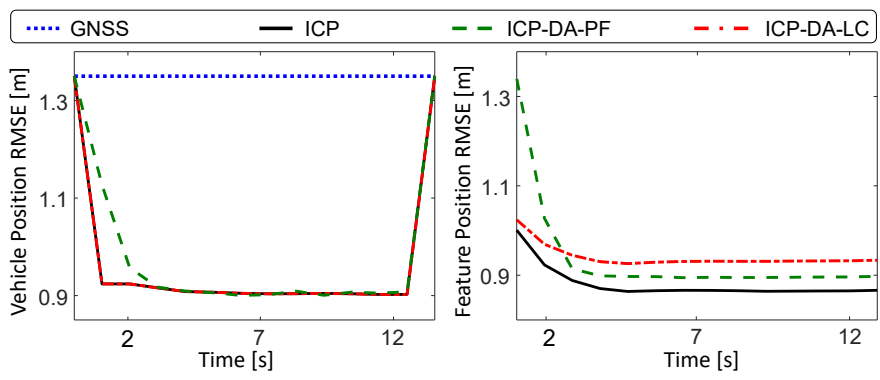

a) $d_{f}=0.5 \mathrm{~m}$
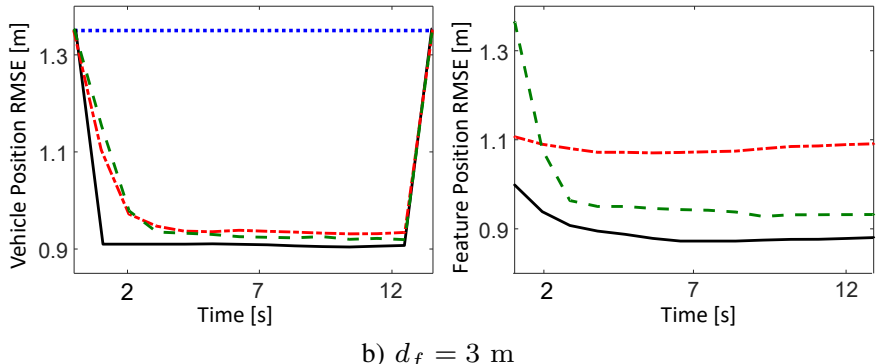

Fig. 7: Vehicle and feature location accuracy versus time for the single-road scenario in Fig. 6 with feature spacing $d_{f}=0.5 \mathrm{~m}$ (a) and $d_{f}=3 \mathrm{~m}$ (b). The ICP-DAPF (orange line) and ICP-DA-LC (green line) methods are compared to the reference GNSS approach (blue dotted line) and the lower bound represented by ICP with known association (black line).

simultaneously sense $N_{f}=2$ static features placed along the sidewalk at a relative distance $d_{f}$ with $\mathrm{V} 2 \mathrm{~F}$ measurement accuracies of $\sigma_{p}^{(\mathrm{V} 2 \mathrm{~F})}=0.2 \mathrm{~m}$ for the position and $\sigma_{v}^{(\mathrm{V} 2 \mathrm{~F})}=0.1 \mathrm{~m} / \mathrm{s}$ for the velocity. The choice of these values is in accordance with the specifications of typical automotive sensors [56].

The performance of the ICP-DA method is assessed using both the PF and LC implementations. Conventional (noncooperative) GNSS and ICP with known data association are simulated as well as benchmarks. For the ICP implementation, the stopping criterion of the iterative BPA-L procedure is obtained by comparing the MMSE location/velocity estimates (for both features and vehicles) with those obtained in the previous iteration. When the difference between the two consecutive estimates is below $10 \mathrm{~cm}$ for the position and below $0.1 \mathrm{~m} / \mathrm{s}$ for the velocity, the iterations are stopped. For the ICP-DA-PF method, the belief of each vehicle and feature is approximated by using $J_{\mathrm{v}}=J_{\mathrm{f}}=5 \cdot 10^{3}$ particles. For ICP-DA-LC, the threshold on the association belief is set to $\eta_{\mathrm{TH}}=1$, meaning that all the $\mathrm{V} 2 \mathrm{~F}$ measurements are paired to the originating features. The performance is evaluated in terms of position/velocity accuracy computed according the Root Mean Square Error (RMSE) of the position/velocity estimate, by averaging over Monte Carlo simulations.

The performance of the localization methods are shown in 
Fig. 7 for feature spacing $d_{f}=0.5 \mathrm{~m}$ (a) and $d_{f}=3 \mathrm{~m}$ (b). For the ICP-DA-LC method, we measured a probability of correct measurement-feature association of, respectively, $54 \%$ and of $92 \%$ in the two cases. The results in Fig. 7-(a) show that for $d_{f}=0.5 \mathrm{~m}$ the ICP-DA-PF and ICP-DA-LC accuracies on vehicles reach the lower bound for known data association, as the errors due to wrong associations are compensated by the proximity of features. The worse performance of the ICPDA-PF method in the first two seconds of V2F sensing is due to the limited number of particles which affects the tracking capability in the transitory. For higher feature inter-distance as in Fig. 7-(b) for $d_{f}=3 \mathrm{~m}$, the association errors decrease the positioning accuracy for both the ICP-DA-PF and ICP-DA-LC methods, with slightly better results for the PF implementation. The performance loss due to the wrong association is more evident on features than on vehicles, as inaccuracies on feature localization have minor impact when they are propagated back to vehicles. We also tested the performance for feature spacing up to $d_{f}=6 \mathrm{~m}$ and we found that above this threshold the association is not critical anymore, as the ICP-DA methods reach the same performance of the ideal ICP with known association, with similar results as in Fig. 7-(a).

From the above results, we can conclude that DA is a crucial issue for cooperative positioning but, if features are close enough (i.e., with spacing comparable with the vehicle localization accuracy) the effect on positioning is negligible. The same is observed for well separated features (i.e., $d_{f}=6 \mathrm{~m}$ in the considered setting) as data association can be easily solved in this case. For intermediate feature spacing (for $d_{f}$ from $3 \mathrm{~m}$ to $6 \mathrm{~m}$ ), a moderate performance loss is observed due to DA errors and PF slightly outperforms LC. Considering the computational complexity, that for ICPDA-PF scales quadratically with the number of particles, the preferred solution is ICP-DA-LC which guarantees both high accuracy and efficiency.

\section{B. Crossroad Scenario with Dynamic Features}

We consider the crossroad scenario of Fig. 1, in which $N_{v}=12$ interconnected vehicles $\left(R_{c}=250 \mathrm{~m}\right)$, grouped in four clusters of three vehicles each, are driving straight ahead along their respective lanes approaching a cross-junction. Spatial coordinates are referred to the center of the junction located in $(0,0)$. The edges of the considered area are placed at $250 \mathrm{~m}$ in any direction from the center, thus each lane is $500 \mathrm{~m}$ long. The vehicle motion is simulated as in (1), with sampling time $T_{s}=1 \mathrm{~s}$ and average velocity of $50 \mathrm{~km} / \mathrm{h}$ along the motion direction. The driving process standard deviations for the longitudinal and lateral directions are set to $\sigma_{a_{i}, \|}^{(\mathrm{V})}=0.35 \mathrm{~m} / \mathrm{s}^{2}$ and $\sigma_{a_{i}, \perp}^{(\mathrm{V})}=0.001 \mathrm{~m} / \mathrm{s}^{2}$, respectively. Then, the uncertainties along the axes $x$ and $y\left(\sigma_{a_{i}, x}^{(\mathrm{V})}\right.$ and $\left.\sigma_{a_{i}, y}^{(\mathrm{V})}\right)$ are defined according to the vehicle motion direction. The GNSS is supposed to provide measurements with the same accuracy over the whole area. Specifically, the GNSS standard deviations are set to $\sigma_{p}^{(\mathrm{V})}=9 \mathrm{~m}$ and $\sigma_{v}^{(\mathrm{V})}=0.25 \mathrm{~m} / \mathrm{s}$ as to simulate a mid-urban environment.

The scenario includes also a set of $N_{f}$ features that are randomly deployed on the sidewalks and sensed by vehi-

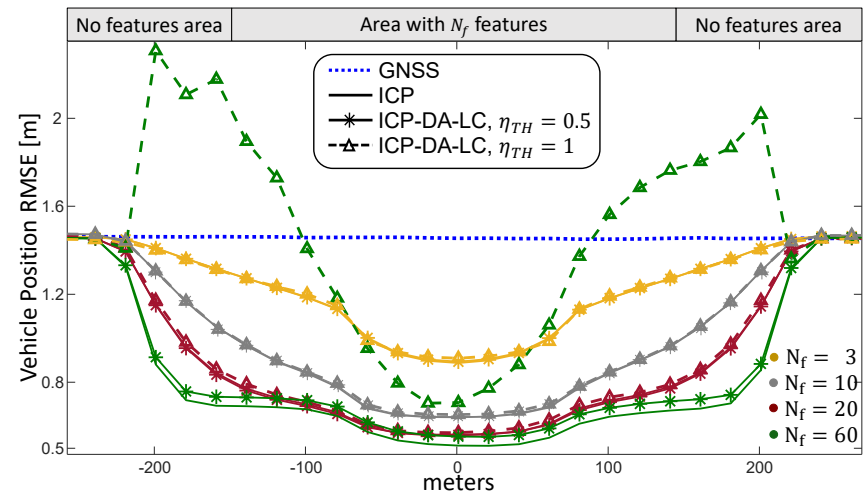

Fig. 8: Vehicle position accuracy versus the distance to the center of the junction for the crossroad scenario in Fig. 1 with static features, for different number of features $N_{f}$. The ICP-DA-LC method (green marked line) is compared to the ICP with known association (red line) and to GNSS-based tracking (blue dotted line).

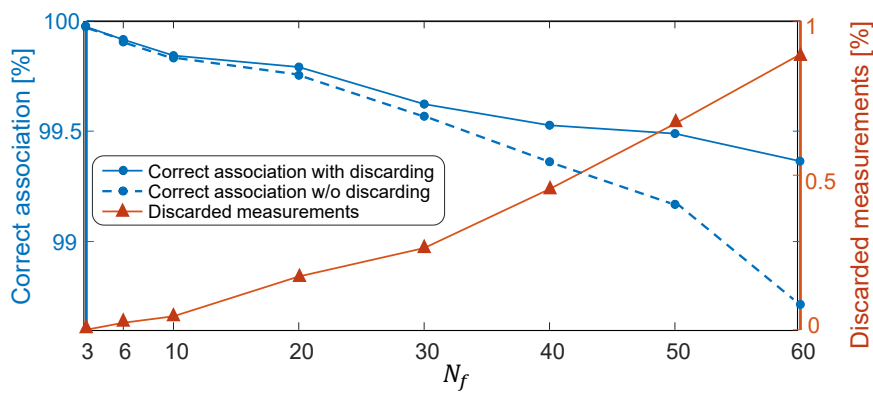

Fig. 9: Percentages of association errors (blue line) and of discarded measurements (orange line) versus the number of features $N_{f}$.

cles in close proximity $\left(R_{s}=75 \mathrm{~m}\right)$. The feature initial location is uniformly distributed within a circle of radius $150 \mathrm{~m}$ centered in $(0,0)$. Feature motion is simulated as in (2), with initial velocity $v_{f}$ and driving process covariance $\mathbf{G}_{k, t}^{(\mathrm{F})}=\mathbf{G} \operatorname{diag}\left(\sigma_{v_{x}}^{(\mathrm{F})^{2}}, \sigma_{v_{y}}^{(\mathrm{F})^{2}}\right) \mathbf{G}^{\mathrm{T}}$, with $\mathbf{G}=\left[T_{s} \mathbf{I}_{2} \mathbf{I}_{2}\right]^{\mathrm{T}}$. As for vehicles, a higher mobility is assumed along the road direction, i.e., the velocity standard deviation in the direction of sidewalk is $\sigma_{v, \|}^{(\mathrm{F})}=\frac{v_{f}}{100} \mathrm{~m} / \mathrm{s}$, while the one in the orthogonal direction is $\sigma_{v, \perp}^{(\mathrm{F})}=\frac{\sigma_{v, \|}^{(\mathrm{F})}}{2} \mathrm{~m} / \mathrm{s}$. Thus, depending on the driving direction of the feature, the velocity uncertainties along the axes $x$ and $y, \sigma_{v, \mathrm{x}}^{(\mathrm{F})}$ and $\sigma_{v, \mathrm{y}}^{(\mathrm{F})}$ respectively, are defined accordingly. The V2F covariance matrix is structured as $\mathbf{R}_{i, \ell, t}^{(\mathrm{V} 2 \mathrm{~F})}=$ $\operatorname{blockdiag}\left(\sigma_{p}^{(\mathrm{V} 2 \mathrm{~F})^{2}} \mathbf{I}_{2}, \sigma_{v}^{(\mathrm{V} 2 \mathrm{~F})^{2}} \mathbf{I}_{2}\right)$, with $\sigma_{p}^{(\mathrm{V} 2 \mathrm{~F})}=0.1 \mathrm{~m}$ and $\sigma_{v}^{(\mathrm{V} 2 \mathrm{~F})}=0.1 \mathrm{~m} / \mathrm{s}$ for each vehicle.

We evaluate the RMSE positioning performance by averaging over 5000 Monte Carlo simulations. Fig. 8 shows the vehicle location accuracy in the proximity of the junction for $N_{f}=\{3,10,20,60\}$ static features. The GNSS positioning accuracy (blue dotted line) is plotted as a reference, while the ICP method with perfect association (continuous line) is the lower bound for the cooperative positioning technique. The proposed ICP-DA-LC method is implemented in two different versions, using for feature detection in (30) the threshold $\eta_{\mathrm{TH}}=0.5$ (i.e., discarding ambiguous $\mathrm{V} 2 \mathrm{~F}$ measurements) and $\eta_{\mathrm{TH}}=1$ (i.e., using all $\mathrm{V} 2 \mathrm{~F}$ measurements). The results in Fig. 8 show a negligible performance loss of the proposed ICP-DA-LC method with respect to the ICP with perfect association. However, it can be noticed that, while for moderate 


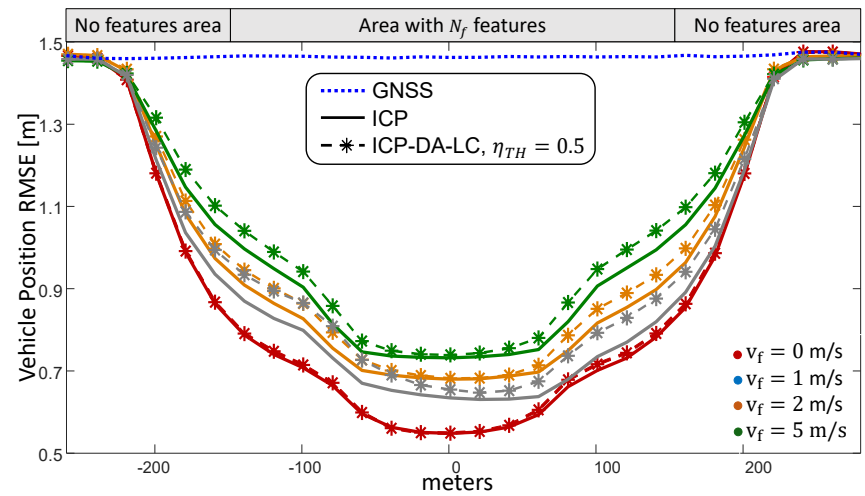

Fig. 10: Vehicle position accuracy versus the distance to the center of the junction for the crossroad scenario in Fig. 1 with $N_{f}=20$ features, for different values of feature velocity $v_{f}=\{1,2,5\} \mathrm{m} / \mathrm{s}$.

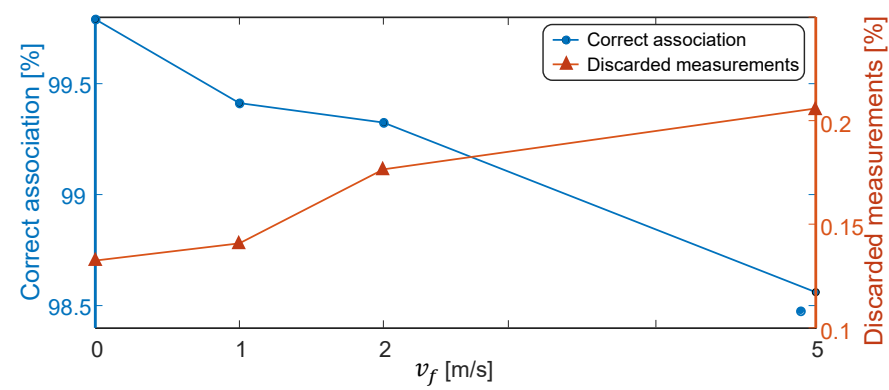

Fig. 11: Percentages of correct association (blue line) and of discarded measurements (orange line) versus the number of feature's velocity $v_{f}$

feature densities $\left(N_{f} \leq 20\right)$ the ICP-DA-LC method without measurement selection does not have significant drawbacks, when features increase and get closer, a selection is required. In fact, for $N_{f}=60$, the vehicle location accuracy of the ICP-DA-LC with $\eta_{\mathrm{TH}}=1$ is even worse than the reference GNSS one, unless in close proximity of the junction, where all vehicles are connected each other and can sense a higher number of features thanks to the road geometry. For $N_{f}=60$, the reduced spacing between features generates ambiguity problems in the association, as proved by the performance loss of ICP-DA-LC with $\eta_{\mathrm{TH}}=1$ compared to ICP with perfect association. Increasing the number of features from $N_{f}=20$ to $N_{f}=60$ does not provide any benefit, as the performance is limited by the association errors.

The probability of correct association for the different values of $N_{f}$, together with the percentage of discarded measurements, is presented in Fig. 9. The figure shows a reduction on the probability of correct association as the number of features increases. This reduction is far more evident for the implementation which uses all measurements $\left(\eta_{\mathrm{TH}}=1\right)$ rather than for the ICP-DA-LC with association selection $\left(\eta_{\mathrm{TH}}=0.5\right)$. In this latter case, in fact, the number of discarded measurements increases with $N_{f}$ and contributes to a significant improvement of the probability of correct association as unreliable measurements (which are likely to be wrongly associated) are discarded.

The results in Fig. 8 and 9 indicate that a moderate number of features, i.e. $N_{f}=20$ corresponding approximately to an average of 4 features simultaneously sensed by each vehicle (this value is obtained considering the selected sensing radius), is enough for the ICP-DA-LC method to significantly outperform the GNSS-based localization. A higher number of features, on the other hand, does not provide any meaningful benefit, as it increases both the probability of erroneous association and the computational complexity. Moreover, the comparable performance obtained by ICP-DALC with measurement selection and ICP with known data association demonstrates that a low-complexity solution avoiding the computational overhead of PF is an efficient approach for vehicular environments.

Fig. 10 illustrates the impact of feature mobility on the ICPDA performance in a scenario with $N_{f}=20$ features moving at an average velocity $v_{f}=\{1,2,5\} \mathrm{m} / \mathrm{s}$. The analysis shows that in case of highly dynamic scenarios (i.e., with feature mobility above the typical pedestrian velocity of $1.5 \mathrm{~m} / \mathrm{s}$ ) the positioning performance degrades but the ICP-DA-LC method with measurement selection is still able to significantly improve the conventional GNSS solution. This conclusion is also confirmed by the analysis of the probability of correct association and measurement discarding in Fig. 11.

\section{Vi. Performance Assessment in a Realistic Urban ARTERIAL SCENARIO}

In this section, we assess by simulation the performance of the ICP-DA method in realistic traffic conditions along an urban arterial in Turin, Italy. We focus on the ICPDA-LC implementation with association selection (i.e., with $\eta_{\mathrm{TH}}=0.5$ ), as this solution was shown to provide the best trade-off between performance and computational complexity in Sec. V. This validation is carried out to analyze the impact on the proposed ICP-DA method of traffic control systems which affect the vehicle distributions over the selected area. In fact, the degree of $\mathrm{V} 2 \mathrm{~V}$ connectivity (and the impact of cooperative sensing) is highly dependent on control strategies which determines the traffic flows and queues at nodes. For this reason, two different traffic conditions are analyzed. Moreover, this analysis in a real urban environment is intended to show that ICP-DA can provide a sub-meter accuracy on vehicle positioning and, thus, it can enable advanced C-ITS services for high levels of automated driving.

\section{A. Traffic Modeling}

We consider the road network in Fig. 12, which includes an urban arterial in Turin crossed by three primary roads and several secondary junctions along approximately $2 \mathrm{~km}$. The area is divided into three zones with different GNSS performance, ranging from open sky to built-up scenarios, namely: two external regions (zone A) characterized by high GNSS accuracy $\sigma_{p}^{(\mathrm{V})}=3 \mathrm{~m}$ and $\sigma_{v}^{(\mathrm{V})}=0.1 \mathrm{~m} / \mathrm{s}$, an intermediate zone B with $\sigma_{p}^{(\mathrm{V})}=8 \mathrm{~m}$ and $\sigma_{v}^{(\mathrm{V})}=0.17 \mathrm{~m} / \mathrm{s}$ and an extreme urban area (zone $\mathrm{C}$ ) with very poor GNSS accuracy $\sigma_{p}^{(\mathrm{V})}=15 \mathrm{~m}$ and $\sigma_{v}^{(\mathrm{V})}=0.45 \mathrm{~m} / \mathrm{s}$. Vehicles randomly enter the road network according to observed traffic demands, and are localized by simulating a GNSS receiver in fast start condition, with prior location belief centered around the true vehicle state, with standard deviations $\sigma_{p}^{(\mathrm{V})}=2 \mathrm{~m}$ 


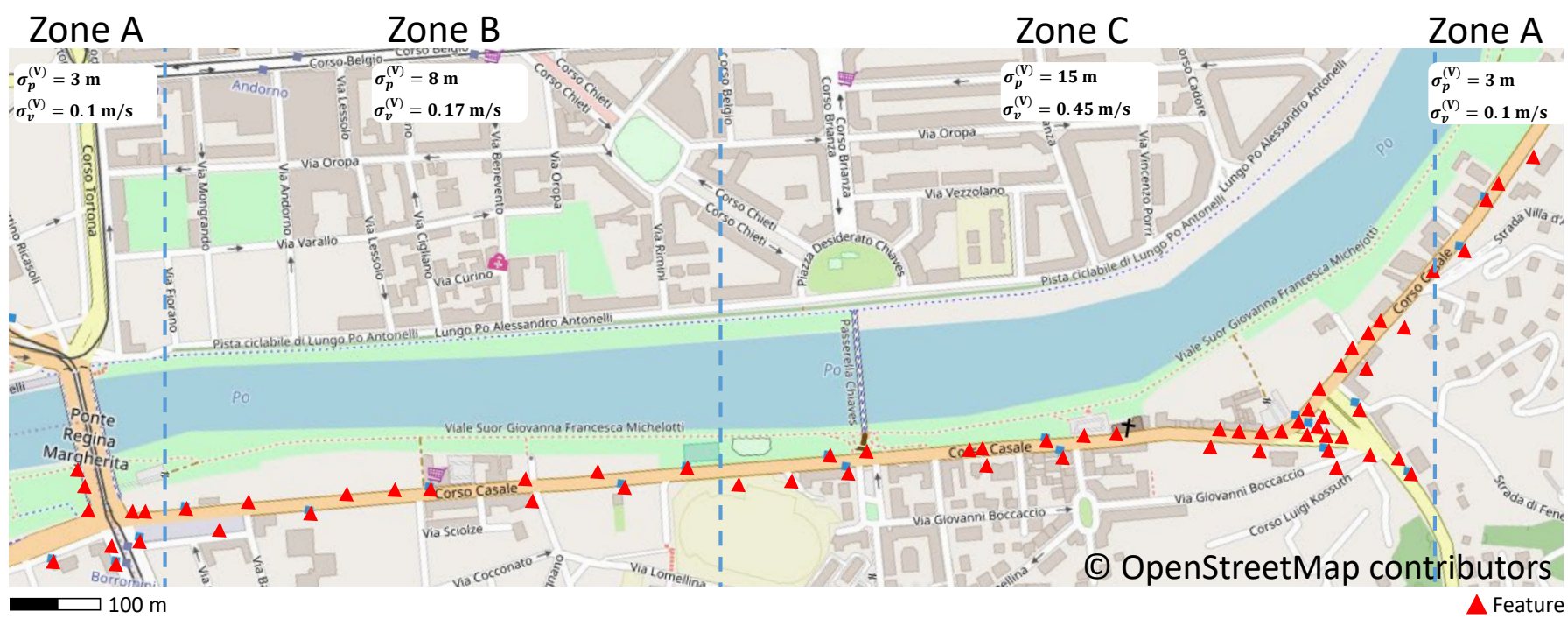

Fig. 12: Urban arterial scenario in Turin, Italy. The area has been divided in three different zones (A, B and C) associated to different GNSS accuracies. Features (red triangles) have been placed along the road infrastructure. Map data copyrighted OpenStreetMap contributors and available from https://www.openstreetmap.org [57].

and $\sigma_{v}^{(\mathrm{V})}=0.1 \mathrm{~m} / \mathrm{s}$. Since the analysis on feature mobility in Sec. V proved that static features are more beneficial for localization enhancement, $N_{f}=64$ static features are considered in correspondence of bus stops, traffic lights, road signs and car parks. The accuracy of feature sensing is set to $\sigma_{p}^{(\mathrm{V} 2 \mathrm{~F})}=0.1 \mathrm{~m}$ and $\sigma_{v}^{(\mathrm{V} 2 \mathrm{~F})}=0.1 \mathrm{~m} / \mathrm{s}$, with a sensing range of $R_{s}=50 \mathrm{~m}$.

Traffic is simulated by using the micro-simulator AIMSUM (Advanced Interactive Microscopic Simulator for Urban and Non-Urban Networks) [58], which updates the vehicles' dynamics every time step of duration $T_{s}=0.75 \mathrm{~s}$. The simulations replicates realistic conditions, including the observed road geometry and traffic control systems at junctions, traffic flows and public transport lines affected by vehicles behaviors and their interactions. To this purpose, the model has been calibrated using real data and describes a common urban scenario with traffic lights affecting the vehicular patterns. The time slot 20:30-21:30 is chosen as reference scenario for traffic demands, which are computed based on real observations of the number of trips performed by the considered vehicle types from origins to destinations. Five traffic control systems regulate the main nodes along the arterial, which is divided in road sections. Each section is composed by one or more lanes according to the vehicle maneuvers detected during the observation phase, as described in [59]. Vehicular traffic along the road sections is simulated by modeling the following components:

1) lane changing for the lateral behavior along the sections,

2) car following for the longitudinal behavior along the sections,

3) traffic control and gap acceptance model for the crossing behavior at nodes.

Traffic control is expected to have a significant impact on the cooperative positioning accuracy. In fact, control strategies tend to form platoons of vehicles to improve the road capacity, thereby they contribute to increase the V2V connectivity and ease the cooperation between vehicles. To investigate the

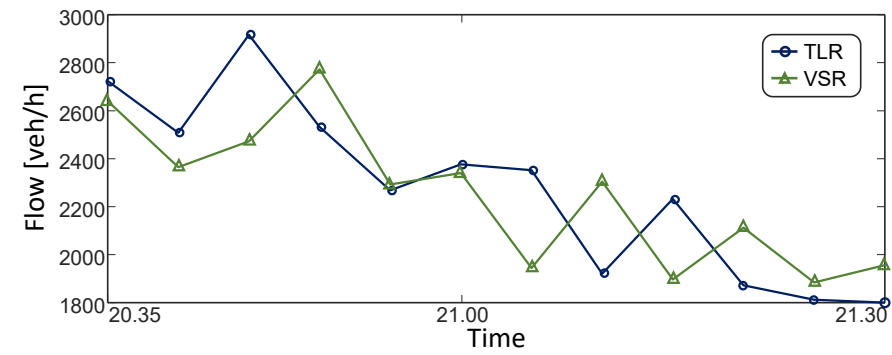

Fig. 13: Traffic flow over time for the two considered traffic conditions: traffic light regulated (TLR) and vehicle self regulated (VSR). Data are aggregated over 5 minutes.

impact of traffic control on the ICP localization techniques, we consider two different scenarios of traffic regulation. The first is a traffic-light regulated (TLR) scenario where intersections are regulated by traffic lights. The second one is a vehicle selfregulated (VSR) scenario, without traffic lights, with vehicles applying gap acceptance models to decide whether they can safely cross the intersection, avoiding conflicts with other vehicles [60]. This second scenario emulates at first approximation an automated driving use-case, as in each time step vehicles autonomously detect other vehicles potentially within the conflicting area, perform computations of the required distance and time parameters, and act during the crossing maneuvers on the base of the gap availability decisions. ${ }^{3}$ The VSR scenario can be considered as representative of a vehicle automated driving system of level 4 (according to the definition in [61]), which can also evolve to a cooperative scenario where safety issues can be improved by V2X applications [62]. In both settings, the flow level is decreasing from the peak-hour to off-peak. Therefore, it is possible to observe over the same simulation a variable saturation rate of the arterial and its impact on the positioning accuracy. The simulated traffic

\footnotetext{
${ }^{3}$ Although automated vehicles have lower reaction times with respect to human drivers, in our experiments we used the same reaction time to compare the two scenarios in the same conditions and focus on the effects of traffic light control.
} 


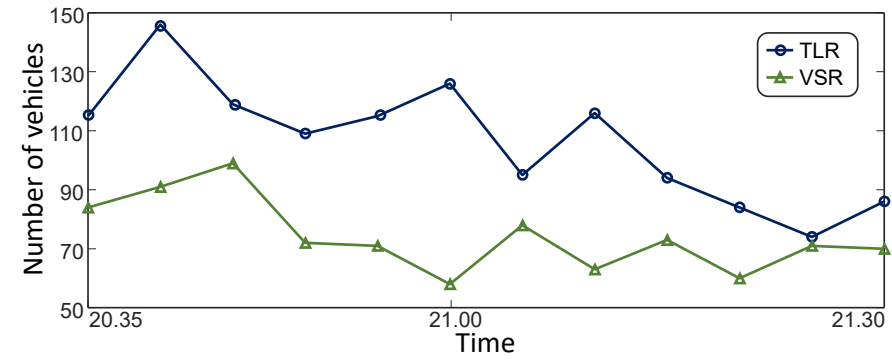

Fig. 14: Number of vehicles inside the scenario for the traffic light regulated (TLR) and vehicle self regulated (VSR) scenarios. Data are aggregated over 5 minutes.

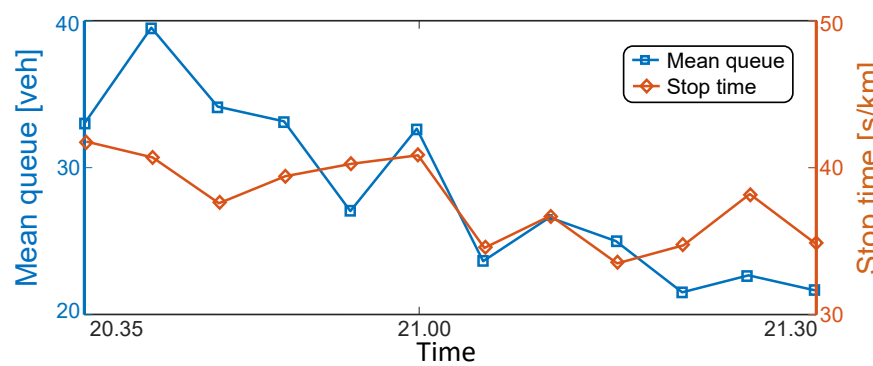

a) Traffic-Light Regulated (TLR) scenario

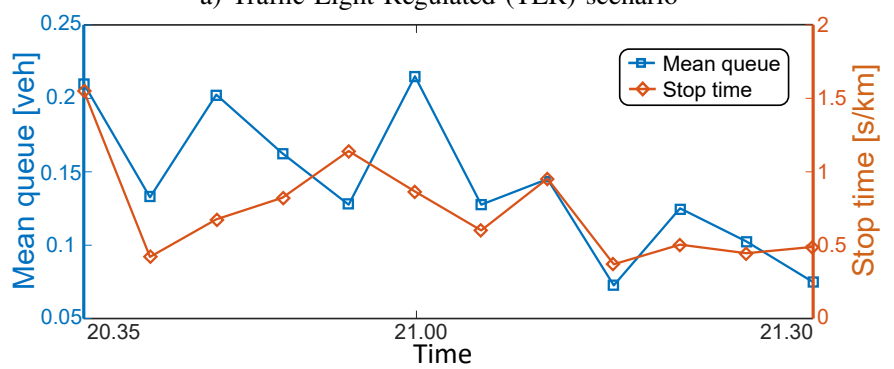

b) Vehicle Self Regulated (VSR) scenario

Fig. 15: Mean queue and stop time for the TLR (a) and VSR (b) scenarios. Data are aggregated over 5 minutes.

conditions are shown in Fig. 13 which plots the total flow exiting from the network in the VSR and TLR scenarios, ranging from about $2800[\mathrm{veh} / \mathrm{h}]$ at the beginning of the considered period to $1800[\mathrm{veh} / \mathrm{h}]$ at the end. This similarity on the output flow trend in the two scenarios is not maintained inside the road network, as expected. As shown in Fig. 14, the number of vehicles inside the network for the TLR scenario is higher with respect to the VSR one. In fact, in the second setting vehicles are more distributed over the arterial and no platooning action is applied. This is confirmed by the results in Figures $15 \mathrm{a}$ and $15 \mathrm{~b}$ which show the mean queue and stop time for, respectively, the TLR and VSR scenarios. In Fig. $15 \mathrm{a}$, the mean queue decreases from almost 40 to 20 vehicles during simulation and the stopped time decreases from $40 \mathrm{~s}$ to $35 \mathrm{~s}$ per $\mathrm{km}$, while in Fig. $15 \mathrm{~b}$ the values are considerably lower. A mean queue less than 1 vehicle and a stop time of approximately $1 \mathrm{~s}$ prove the reduction on the number of vehicles inside the arterial.

These two different traffic conditions create two completely independent vehicular network scenarios with different V2V and V2F connections. This diversity is compared in the next section to assess the performance of the proposed cooperative positioning method.

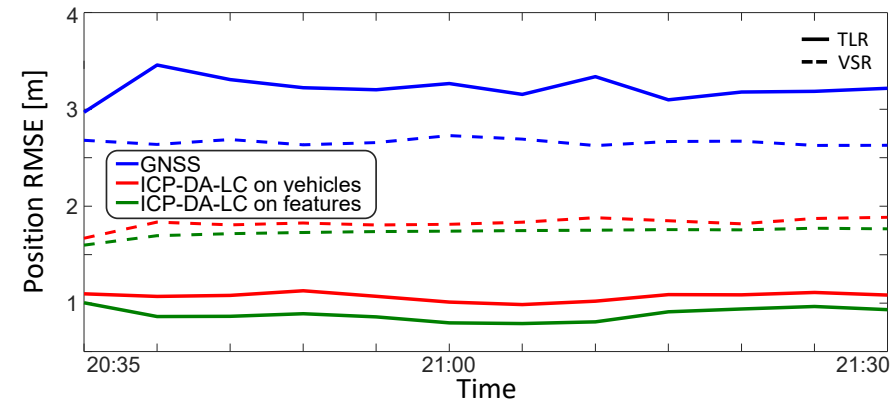

a) Position

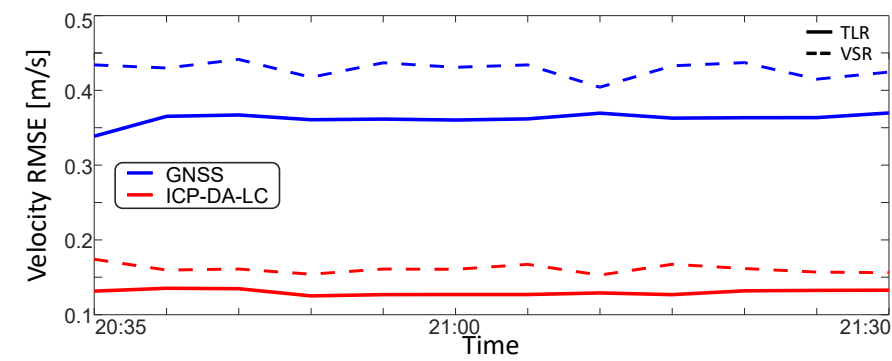

b) Velocity

Fig. 16: Averaged accuracy of position (a) and velocity (b) over time for the two considered scenarios: TLR (continuous line) and VSR (dashed line). The GNSS-based accuracy on vehicles (blue line) is taken as reference for the ICP-DA-LC estimate on vehicle position (red line). The green line in (a) indicates the accuracy on features.

\section{B. Performance Analysis}

In the following, we compare the performance of the ICPDA-LC method with the conventional GNSS-based tracking in both the TLR and VSR traffic scenarios. In Fig. 16, the aggregate RMSE (with aggregation interval of 5 minutes) of the vehicle/feature state estimates, is plotted versus time for the two different traffic conditions, i.e. the TLR scenario (continuous line) and the VSR one (dashed line). The RMSE has been obtained by averaging over vehicles and features. The top figure (Fig. 16a) is referred to vehicle position, while the bottom one (Fig. 16b) is dedicated to the vehicle velocity. Blue and red lines denote the accuracy of vehicle tracking for, respectively, the GNSS and ICP-DA-LC methods, while the green line indicates the ICP-DA-LC accuracy on features.

The results in Fig. 16a prove that the ICP-DA-LC method outperforms the conventional GNSS system over the whole simulation time, thanks to the dense $\mathrm{V} 2 \mathrm{~V}$ connectivity that eases the cooperation in the urban area. The improvement is far more relevant in the TLR scenario (continuous line) than in the VSR one (dashed line), due to the higher number of vehicles and the continuous refinement of the feature estimates performed by vehicles during the stop periods. On the other hand, the average GNSS tracking accuracy is better in VSR than TLR, as vehicles do not stop at the numerous intersections of the built-up zone $\mathrm{C}$ and thus spend less time in this area with highly degraded GNSS signals. For the cooperative approach, Fig. 16a shows similar accuracy results for vehicles and features, confirming that the precise localization of features obtained by the V2V cooperation directly reflects on the vehicle location accuracy. In the ideal case of perfectly localized features, the ICP method behaves as an augmented 


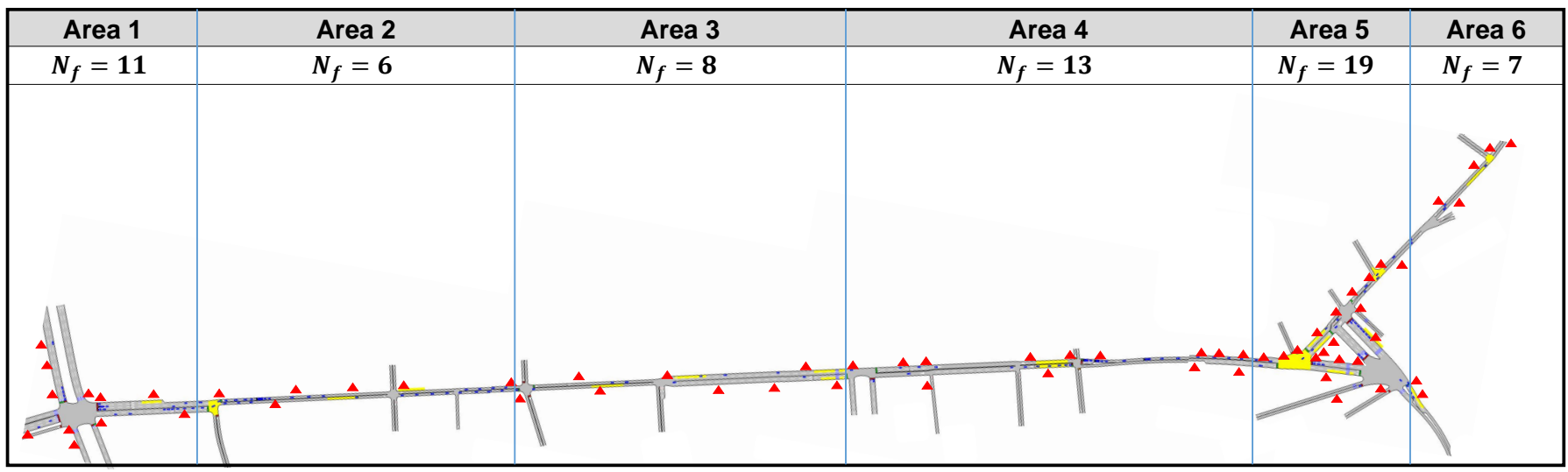

a) Area of analysis of the Turin traffic scenario
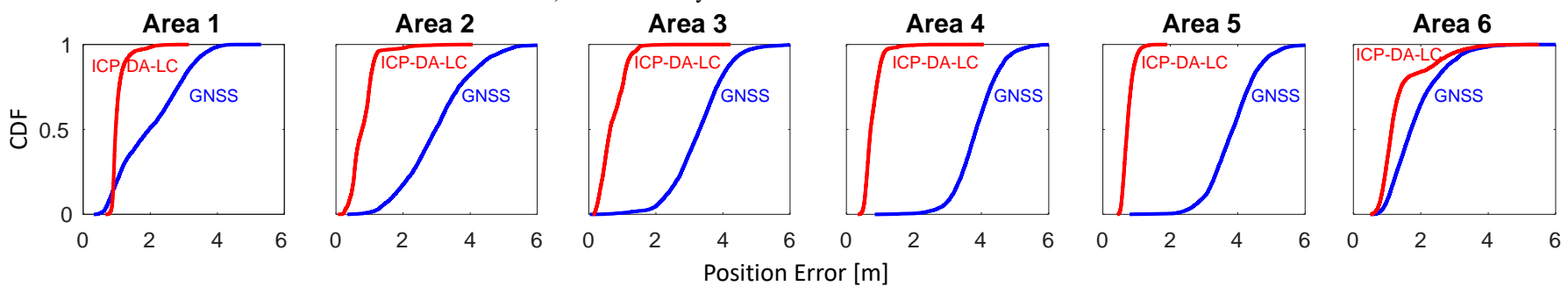

b) $\mathrm{CDF}$ of the vehicle position accuracy

Fig. 17: (a) Map of the Turin road network divided into six areas, characterized by different densities of features and GNSS conditions, with highlighted locations of vehicles (blue dots) and features (red triangles). (b) CDF of vehicle position accuracy observed in the in TLR scenario over the different areas.

positioning system with many fixed anchors (i.e., the features) along the road infrastructure.

The analysis on the location estimate accuracy is extended to the velocity in Fig. 16b. Again, the performance of the ICP-DA-LC method is better in the regulated scenario than in the self-regulated one. This now holds also for the GNSS method, as the length of time the vehicle remains stationary at junctions allows the average of the observations to be taken which improves the accuracy of the velocity estimate.

A deeper analysis of the location accuracy over the space domain is carried out in Fig. 17a for the TLR traffic scenario. The road network is divided into six areas characterized by different GNSS accuracies and feature densities, as indicated in Fig. 17a. The Cumulative Distribution Function (CDF) of the vehicle position accuracy averaged over the whole simulation time is computed for each area in Fig. 17b. The performance augmentation provided by the ICP-DA-LC method is significantly different from one area to the other. In particular, a meaningful gain is obtained in the critical built-up areas 4 and 5, while minor improvements are observed in areas 1 and 6 where the GNSS tracking is already highly reliable. Specifically, the highest improvement in localization is in Area 5 , where the CDF at $95 \%$ of confidence for the GNSS solution indicates a vehicle position error of $5 \mathrm{~m}$, while the ICP-DA is able to improve this value to $1 \mathrm{~m}$.

The performance analysis over the time domain is in Fig. 18, which presents the vehicle location accuracy versus time, for the along-track and the cross-track directions in areas 2 (Fig. 18a) and 5 (Fig. 18b). Numerical results show that the ICPDA-LC method is able to provide a sub-meter level accuracy in both along-track and cross-track directions, compensating the

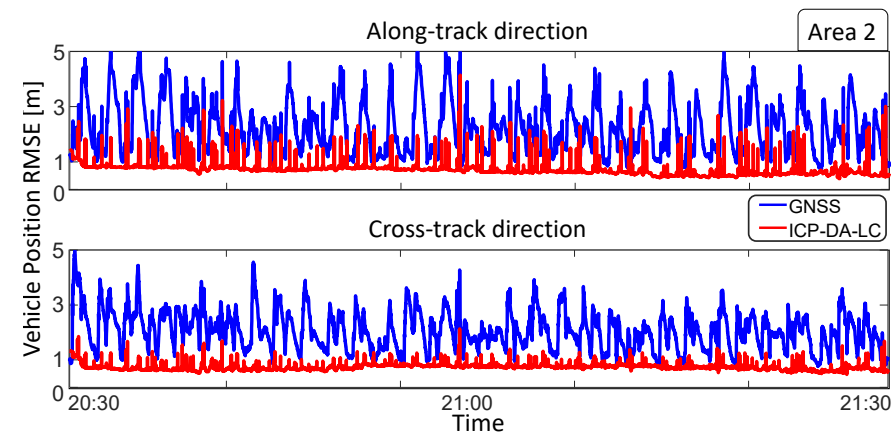

a) Area 2

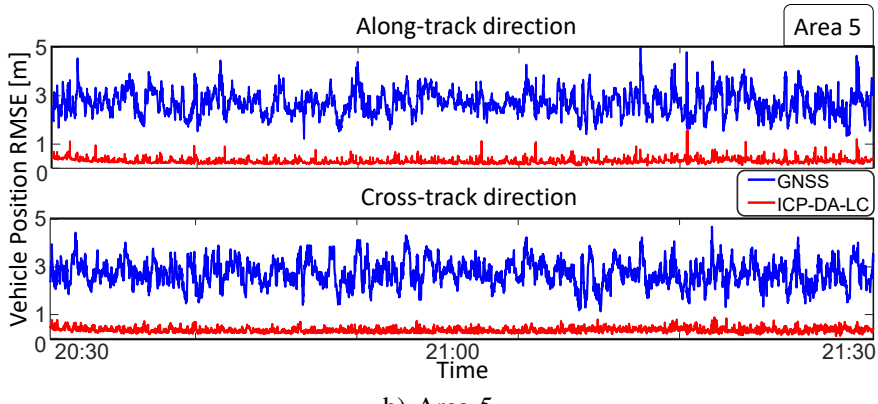

b) Area 5

Fig. 18: Vehicle location accuracy over time for both the along-track and cross-track directions in areas 2 (a) and 5 (b) for the TLR scenario.

degradation introduced by multipath and non-line-of-sight of the GNSS signals. The large number of sensed features along with a road configuration that facilitates, with its numerous intersections, the formation of platoons of cooperative vehicles continuously refining their estimates on the surrounding features, has an extremely positive impact on the localization performance. Furthermore, the figure highlights a marked peri- 


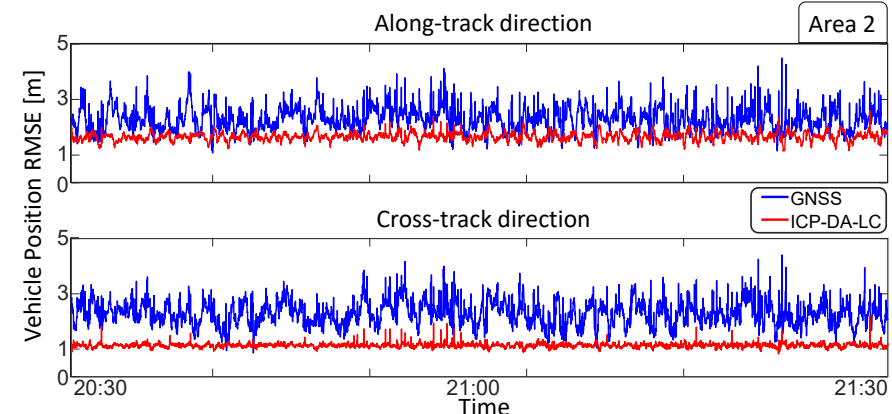

Fig. 19: Vehicle location accuracy over time for both the along-track and cross-track directions in area 2 for the VSR scenario.

odicity of the GNSS accuracy pattern over time in Area 2, due to the cyclic regulation of the traffic flows by the sequence of traffic lights along the arterial. In fact, during stop phases vehicles can refine their positioning accuracy by time averaging. The ICP-DA-LC method, on the other hand, provides a more stable performance over time, as the accuracy benefits from space averaging thanks to the cooperation between spatially distributed vehicles. This equalization effect is more evident in the cross-track direction, as expected. This important remark, along with the high precision provided by ICP-DA-LC (below $1 \mathrm{~m})$, makes the proposed method a reliable solution for lane detection in urban contexts. Referring, for example, to the basic set of applications enabling the advanced ITS services defined by ETSI [63], the ICP approach could be used to meet the challenging performance requirements in terms of location accuracy.

Finally, Fig. 19 presents a similar analysis for the VSR scenario in area 2 . In this case, the GNSS estimate is no more affected by traffic lights and the localization accuracy pattern is non periodic. The overall average accuracy is worse than in the TLR scenario for both GNSS and ICP-DA-LC, but the performance gain of the proposed cooperative method is remarkable especially in the cross-track direction.

The above analysis confirms that the diffusion of smart vehicles with advanced driver assistance systems, combining onboard ranging sensors with $\mathrm{V} 2 \mathrm{X}$ communication technologies, can significantly improve vehicle positioning in urban areas thanks to cooperative strategies for perception and localization. This enhanced positioning is expected to enable new innovative C-ITS services especially in emerging automated driving scenarios, where the formation of tight convoys or platoons of vehicles facilitate the cooperation process. Examples of services include high-precision traffic monitoring, lane-change assistance, infractions detection, inter-vehicle communication efficiency and so forth.

\section{CONCLUSIONS}

In this paper, we developed a new distributed Bayesian framework for cooperative data association and vehicle localization in urban traffic scenarios. The analysis has been carried out considering smart vehicles equipped with on-board ranging sensors and V2X communication devices, as envisioned in the C-ITS context. We proposed two new ICP techniques, namely the ICP-DA-PF method based on a distributed PF solution to handle the non-Gaussian location statistics and the ICPDA-LC method that simplifies the problem to a Gaussian setting and employs a distributed KF approach to reduce the communication/processing overhead at vehicles. The ICP-DALC method was shown to provide the best trade-off between complexity and accuracy, closely attaining the performance of the ICP method with known association.

Performance results in realistic traffic scenarios confirmed that the proposed ICP-DA methods can significantly improve the GNSS performance reaching a sub-metric accuracy. The investigation on the DA performance highlighted the importance of feature selection mechanisms, to avoid association errors that could degrade the cooperative positioning performance. Quasi-stationary features have to be preferred as they can be localized with higher accuracy acting as virtual anchors for the positioning process.

Traffic flow micro-simulation over a real urban network showed how traffic control systems affect the distribution of vehicles over the roads and impact on the graph connectivity for inter-vehicle cooperation. In particular, a high density of vehicles and features, as well as the formation of vehicle platoons as induced by traffic-light systems (or, in perspective, by cooperative maneuvering strategies) guarantees a high degree of $\mathrm{V} 2 \mathrm{~V}$ connectivity and increases the accuracy of the cooperative sensing method. The analysis also showed that in urban scenarios the vehicle dynamics and the severe degradation of satellite signals induce high fluctuations of the GNSS tracking over space and time, while the ICP-DA method is capable to mitigate this non-stationary behavior, reducing the impact of external factors on the localization performance.

The proposed technique thus emerges as a promising solution to enhance both the accuracy and the robustness of positioning in urban scenarios. This enhancement is expected to enable new innovative C-ITS services especially in emerging automated driving scenarios, where the formation of tight convoys or platoons of vehicles facilitate the cooperation process.

\section{APPENDIX A}

According to the Bayesian rule [64], the joint posterior pdf of the association and the vehicle-feature states is:

$p\left(\boldsymbol{\theta}_{1: t}, \boldsymbol{\alpha}_{1: t}, \boldsymbol{\beta}_{1: t} \mid \boldsymbol{z}_{1: t}\right) \propto p\left(\boldsymbol{z}_{1: t} \mid \boldsymbol{\theta}_{1: t}, \boldsymbol{\alpha}_{1: t}, \boldsymbol{\beta}_{1: t}\right) p\left(\boldsymbol{\theta}_{1: t}, \boldsymbol{\alpha}_{1: t}, \boldsymbol{\beta}_{1: t}\right)$,

where the likelihood function is $p\left(\boldsymbol{z}_{1: t} \mid \boldsymbol{\theta}_{1: t}, \boldsymbol{\alpha}_{1: t}, \boldsymbol{\beta}_{1: t}\right)=$ $p\left(\boldsymbol{z}_{1: t}^{(\mathrm{V})} \mid \mathbf{x}_{1: t}^{(\mathrm{V})}\right) p\left(\boldsymbol{z}_{1: t}^{(\mathrm{V} 2 \mathrm{~F})} \mid \boldsymbol{\theta}_{1: t}, \boldsymbol{\alpha}_{1: t}\right)$ as the measurements (3)-(4) are conditionally independent. Assuming also the measurements as independent over time and vehicles, and the V2F observations as independent over features, the likelihood can be factorized as:

$$
\begin{aligned}
p\left(\boldsymbol{z}_{1: t} \mid \boldsymbol{\theta}_{1: t}, \boldsymbol{\alpha}_{1: t}\right) & =\prod_{t^{\prime}=1}^{t} \prod_{i=1}^{N_{v}} p\left(\boldsymbol{z}_{i, t^{\prime}}^{(\mathrm{V})} \mid \mathbf{x}_{i, t^{\prime}}^{(\mathrm{V})}\right) \times \\
& \prod_{k=1}^{N_{f}} p\left(\boldsymbol{z}_{i, t^{\prime}}^{(\mathrm{V} 2 \mathrm{~F})} \mid \mathbf{x}_{i, t^{\prime}}^{(\mathrm{V})}, \mathbf{x}_{k, t^{\prime}}^{(\mathrm{F})}, \boldsymbol{\alpha}_{i, k, t^{\prime}}\right) .
\end{aligned}
$$

Moreover, assuming the observation-feature association as independent over time and from vehicle/feature states, 
the prior pdf in (32) simplifies to $p\left(\boldsymbol{\theta}_{1: t}, \boldsymbol{\alpha}_{1: t}, \boldsymbol{\beta}_{1: t}\right)=$ $p\left(\boldsymbol{\alpha}_{1: t}, \boldsymbol{\beta}_{1: t}\right) p\left(\boldsymbol{\theta}_{1: t}\right)$ with, according to (8),

$$
p\left(\boldsymbol{\alpha}_{1: t}, \boldsymbol{\beta}_{1: t}\right) \propto \prod_{t^{\prime}=1}^{t} \prod_{i=1}^{N_{v}} \prod_{k=1}^{N_{f}} \prod_{\ell=1}^{O_{i, t^{\prime}}} \Psi\left(\alpha_{i, k, t}, \beta_{i, \ell, t}\right) .
$$

Recalling that vehicle and feature states evolve independently according to Markovian dynamic models (1) and (2), the prior pdf of the overall vehicle/feature states factorizes as:

$$
\begin{aligned}
p\left(\boldsymbol{\theta}_{1: t}\right)= & \left(\prod_{i=1}^{N_{v}} p\left(\mathbf{x}_{i, 0}^{(\mathrm{V})}\right) \prod_{t^{\prime}=1}^{t} p\left(\mathbf{x}_{i, t^{\prime}}^{(\mathrm{V})} \mid \mathbf{x}_{i, t^{\prime}-1}^{(\mathrm{V})}\right)\right) \times \\
& \left(\prod_{k=1}^{N_{f}} p\left(\mathbf{x}_{k, 0}^{(\mathrm{F})}\right) \prod_{t^{\prime \prime}=1}^{t} p\left(\mathbf{x}_{k, t^{\prime \prime}}^{(\mathrm{F})} \mid \mathbf{x}_{k, t^{\prime \prime}-1}^{(\mathrm{F})}\right)\right),
\end{aligned}
$$

where $p\left(\mathbf{x}_{i, 0}^{(\mathrm{V})}\right)$ and $p\left(\mathbf{x}_{k, 0}^{(\mathrm{F})}\right)$ denote the prior pdfs at time $t=0$.

Considering (33), (34) and (35), the factorization of (32) is given by (9).

\section{REFERENCES}

[1] "IEEE Standard for Information technology- Local and metropolitan area networks- Specific requirements- Part 11: Wireless LAN Medium Access Control (MAC) and Physical Layer (PHY) Specifications Amendment 6: Wireless Access in Vehicular Environments," IEEE Std 802.11p-2010 (Amendment to IEEE Std 802.11-2007 as amended by IEEE Std 802.11k-2008, IEEE Std 802.11r-2008, IEEE Std 802.11y2008, IEEE Std 802.11n-2009, and IEEE Std 802.11w-2009), pp. 1-51, Jul. 2010.

[2] "Intelligent Transport Systems (ITS); Vehicular Communications; Basic Set of Applications; Part 2: Specification of Cooperative Awareness Basic Service," ETSI EN 302 637-2, Nov. 2014.

[3] "Study on LTE support for Vehicle-to-Everything (V2X) services," 3GPP TR 22.885, Release 14, Mar. 2015.

[4] H. Wymeersch, G. Seco-Granados, G. Destino, D. Dardari, and F. Tufvesson, "5G mmWave Positioning for Vehicular Networks," IEEE Wireless Comm., vol. 24, no. 6, pp. 80-86, Dec. 2017.

[5] L. Kong, M. K. Khan, F. Wu, G. Chen, and P. Zeng, "Millimeter-Wave Wireless Communications for IoT-Cloud Supported Autonomous Vehicles: Overview, Design, and Challenges," IEEE Comm. Mag., vol. 55, no. 1, pp. 62-68, Jan. 2017.

[6] L. Chen and C. Englund, "Cooperative ITS - EU Standards to Accelerate Cooperative Mobility," in 2014 Int. Conf. Connected Veh. and Expo, Nov. 2014, pp. 681-686.

[7] H. Wymeersch, G. R. de Campos, P. Falcone, L. Svensson, and E. G. Strm, "Challenges for Cooperative ITS: Improving Road Safety Through the Integration of Wireless Communications, Control, and Positioning," in 2015 Int. Conf. Comput. Netw. and Comm., Feb. 2015, pp. 573-578.

[8] L. Hobert, A. Festag, I. Llatser, L. Altomare, F. Visintainer, and A. Kovacs, "Enhancements of V2X Communication in Support of Cooperative Autonomous Driving," IEEE Comm. Mag., vol. 53, no. 12, pp. 64-70, Dec. 2015.

[9] M. Gerla, E. K. Lee, G. Pau, and U. Lee, "Internet of Vehicles: From Intelligent Grid to Autonomous Cars and Vehicular Clouds," in 2014 IEEE World Forum Internet Things, Mar. 2014, pp. 241-246.

[10] S. Kato, S. Tsugawa, K. Tokuda, T. Matsui, and H. Fujii, "Vehicle Control Algorithms for Cooperative Driving with Automated Vehicles and Intervehicle Communications," IEEE Trans. Intell. Transp. Syst., vol. 3, no. 3, pp. 155-161, Sep. 2002.

[11] S. Kim and W. Liu, "Cooperative Autonomous Driving: A Mirror Neuron Inspired Intention Awareness and Cooperative Perception Approach," IEEE Intell. Transp. Syst. Mag, vol. 8, no. 3, pp. 23-32, Jul. 2016.

[12] M. During and K. Lemmer, "Cooperative Maneuver Planning for Cooperative Driving," IEEE Intell. Transp. Syst. Mag., vol. 8, no. 3, pp. 8-22, Jul. 2016.

[13] G. Pocovi, M. Lauridsen, B. Soret, K. I. Pedersen, and P. Mogensen, "Automation for On-Road Vehicles: Use Cases and Requirements for Radio Design," in 2015 IEEE 82nd Veh. Technol. Conf., Sep. 2015.

[14] 5GPPP, "5G Automotive Vision," Oct. 2015.
[15] D. Caveney, "Cooperative Vehicular Safety Applications," IEEE Control Syst., vol. 30, no. 4, pp. 38-53, Aug. 2010.

[16] S. E. Shladover and S.-K. Tan, "Analysis of Vehicle Positioning Accuracy Requirements for Communication-Based Cooperative Collision Warning," J. Intell. Transp. Syst, vol. 10, no. 3, pp. 131-140, 2006.

[17] E. D. Kaplan and C. Hegarty, Understanding GPS: Principles and Applications. Norwood: Artech House, 2006.

[18] A. Küpper, Location-Based Services: Fundamentals and Operation. Wiley, 2005.

[19] M. W. M. G. Dissanayake, P. Newman, S. Clark, H. F. Durrant-Whyte, and M. Csorba, "A Solution to the Simultaneous Localization and Map Muilding (SLAM) problem," IEEE Trans. Robot. Autom., vol. 17, no. 3, pp. 229-241, Jun. 2001.

[20] G. Bresson, Z. Alsayed, L. Yu, and S. Glaser, "Simultaneous Localization and Mapping: A Survey of Current Trends in Autonomous Driving," IEEE Trans. Intell. Veh., vol. 2, no. 3, pp. 194-220, Sep. 2017.

[21] C. S. Lee, D. E. Clark, and J. Salvi, "SLAM With Dynamic Targets via Single-Cluster PHD Filtering," IEEE J. Sel. Topics Signal Process., vol. 7, no. 3, pp. 543-552, Jun. 2013.

[22] J. Levinson and S. Thrun, "Robust Vehicle Localization in Urban Environments Using Probabilistic Maps," in 2010 IEEE Int. Conf. Robot. Autom., May 2010, pp. 4372-4378.

[23] K. lassoued, P. Bonnifait, and I. Fantoni, "Cooperative Localization with Reliable Confidence Domains Between Vehicles Sharing GNSS Pseudoranges Errors with No Base Station," IEEE Intell. Transp. Syst. Mag., vol. 9, no. 1, pp. 22-34, Jan 2017.

[24] J. Liu, B. G. Cai, and J. Wang, "Cooperative Localization of Connected Vehicles: Integrating GNSS With DSRC Using a Robust Cubature Kalman Filter," IEEE Trans. Intell. Transp. Syst., vol. 18, no. 8, pp. 2111-2125, Aug. 2017.

[25] G. M. Hoang, B. Denis, J. Hrri, and D. T. M. Slock, "Cooperative Localization in GNSS-aided VANETs with Accurate IR-UWB Range Measurements," in 2016 13th Workshop Positioning Navig. and Comm., Oct. 2016, pp. 1-6.

[26] N. Alam, A. T. Balaei, and A. G. Dempster, "Relative Positioning Enhancement in VANETs: A Tight Integration Approach," IEEE Trans. Intell. Transp. Syst., vol. 14, no. 1, pp. 47-55, Mar. 2013.

[27] M. Rohani, D. Gingras, and D. Gruyer, "A Novel Approach for Improved Vehicular Positioning Using Cooperative Map Matching and Dynamic Base Station DGPS Concept," IEEE Trans. Intell. Transp. Syst., vol. 17, no. 1, pp. 230-239, Jan. 2016.

[28] H. Li and F. Nashashibi, "Cooperative Multi-Vehicle Localization Using Split Covariance Intersection Filter," IEEE Intell. Transp. Syst. Mag., vol. 5, no. 2, pp. 33-44, Apr. 2013.

[29] J. Yao, A. T. Balaei, M. Hassan, N. Alam, and A. G. Dempster, "Improving Cooperative Positioning for Vehicular Networks," IEEE Trans. Veh. Technol., vol. 60, no. 6, pp. 2810-2823, Jul. 2011.

[30] S. Fujii, A. Fujita, T. Umedu, S. Kaneda, H. Yamaguchi, T. Higashino, and M. Takai, "Cooperative Vehicle Positioning via V2V Communications and Onboard Sensors," in 2011 IEEE Veh. Technol. Conf., Sep. 2011, pp. 1-5.

[31] N. M. Drawil and O. Basir, "Intervehicle-Communication-Assisted Localization," IEEE Trans. Intell. Transp. Syst., vol. 11, no. 3, pp. 678-691, Sep. 2010.

[32] S. Severi, h. Wymeersch, J. Hrri, M. Ulmschneider, B. Denis, and M. Bartels, "Beyond GNSS: Highly Accurate Localization for Cooperative-Intelligent Transport Systems," in 2018 IEEE Wireless Comm. Netw. Conf., Apr. 2018.

[33] S. Kuutti, S. Fallah, K. Katsaros, M. Dianati, F. Mccullough, and A. Mouzakitis, "A Survey of the State-of-the-Art Localization Techniques and Their Potentials for Autonomous Vehicle Applications," IEEE Internet Things J., vol. 5, no. 2, pp. 829-846, Apr. 2018.

[34] N. Alam and A. G. Dempster, "Cooperative Positioning for Vehicular Networks: Facts and Future," IEEE Trans. Intell. Transp. Syst., vol. 14, no. 4, pp. 1708-1717, Dec. 2013.

[35] M. Rohani, D. Gingras, V. Vigneron, and D. Gruyer, "A New Decentralized Bayesian Approach for Cooperative Vehicle Localization Based on Fusion of GPS and VANET Based Inter-Vehicle Distance Measurement," IEEE Intell. Transp. Syst. Mag., vol. 7, no. 2, pp. 85-95, Jun. 2015.

[36] R. Parker and S. Valaee, "Vehicular Node Localization Using ReceivedSignal-Strength Indicator," IEEE Trans. Veh. Technol., vol. 56, no. 6, pp. 3371-3380, Nov. 2007

[37] A. Fascista, G. Ciccarese, A. Coluccia, and G. Ricci, "Angle of ArrivalBased Cooperative Positioning for Smart Vehicles," IEEE Trans. Intell. Transp. Syst., pp. 1-13, Nov. 2017. 
[38] N. Alam, A. T. Balaei, and A. G. Dempster, "A DSRC Doppler-Based Cooperative Positioning Enhancement for Vehicular Networks With GPS Availability," IEEE Trans. Veh. Technol., vol. 60, no. 9, pp. 4462-4470, Nov. 2011.

[39] G. Soatti, M. Nicoli, N. Garcia, B. Denis, R. Raulefs, and H. Wymeersch, "Enhanced Vehicle Positioning in Cooperative ITS by Joint Sensing of Passive Features," in 2017 IEEE 20th Int. Conf. Intell. Transp. Syst., Oct. 2017, pp. 1-6.

[40] — "Implicit Cooperative Positioning in Vehicular Networks," IEEE Trans. Intell. Transp. Syst., pp. 1-17, 2018.

[41] F. Meyer, O. Hlinka, H. Wymeersch, E. Riegler, and F. Hlawatsch, "Distributed Localization and Tracking of Mobile Networks Including Noncooperative Objects," IEEE Trans. Signal Inf. Process. Netw., vol. 2, no. 1, pp. 57-71, Mar. 2016.

[42] F. Meyer, T. Kropfreiter, J. L. Williams, R. Lau, F. Hlawatsch, P. Braca, and M. Z. Win, "Message Passing Algorithms for Scalable Multitarget Tracking," Proc. IEEE, vol. 106, no. 2, pp. 221-259, Feb. 2018.

[43] F. Meyer, P. Braca, P. Willett, and F. Hlawatsch, "A Scalable Algorithm for Tracking an Unknown Number of Targets Using Multiple Sensors," IEEE Trans. Signal Process., vol. 65, no. 13, pp. 3478-3493, Jul. 2017.

[44] J. Williams and R. Lau, "Approximate Evaluation of Marginal Association Probabilities with Belief Propagation," IEEE Trans. Aerosp. Electron. Syst., vol. 50, no. 4, pp. 2942-2959, Oct. 2014.

[45] M. Frohle, C. Lindberg, and H. Wymeersch, "Cooperative Localization of Vehicles without Inter-Vehicle Measurements," in 2018 IEEE Wireless Comm. Netw. Conf., Apr. 2018.

[46] F. R. Kschischang, B. J. Frey, and H. A. Loeliger, "Factor Graphs and the Sum-Product Algorithm," IEEE Trans. Inf. Theory, vol. 47, no. 2, pp. 498-519, Feb. 2001.

[47] R. Olfati-Saber, J. A. Fax, and R. M. Murray, "Consensus and Cooperation in Networked Multi-Agent Systems," Proc. IEEE, vol. 95, no. 1, pp. 215-233, Jan. 2007.

[48] M. Brambilla, G. Soatti, and M. Nicoli, "Precise Vehicle Positioning by Cooperative Feature Association and Tracking in Vehicular Networks," in 2018 IEEE Statistic. Signal Process. Workshop, Jun. 2018.

[49] V. Astarita, V. Giofré, G. Guido, and A. Vitale, "The Use of Adaptive Traffic Signal Systems Based on Floating Car Data," Wireless Comm. and Mobile Comput., vol. 2017, Dec. 2017.

[50] "Exploring GLOSA Systems in the Field: Technical Evaluation and Results," Computer Comm., vol. 120, pp. 112 - 124, May 2018.

[51] X. Rong Li and V. P. Jilkov, "Survey of Maneuvering Target Tracking. Part I. Dynamic Models," IEEE Trans. Aerosp. Electron. Syst., vol. 39, no. 4, pp. 1333-1364, Oct. 2003.

[52] F. Gustafsson and F. Gunnarsson, "Mobile Positioning Using Wireless Networks: Possibilities and Fundamental Limitations based on Available Wireless Network Measurements," IEEE Signal Process. Mag., vol. 22, no. 4, pp. 41-53, Jul. 2005.

[53] F. Meyer, P. Braca, P. Willett, and F. Hlawatsch, "Scalable multitarget tracking using multiple sensors: A belief propagation approach," in 2015 18th Int. Conf. Inf. Fusion, Jul. 2015.

[54] H. A. Loeliger, "An Introduction to Factor Graphs," IEEE Signal Process. Mag., vol. 21, no. 1, pp. 28-41, Jan. 2004.

[55] M. S. Arulampalam, S. Maskell, N. Gordon, and T. Clapp, "A Tutorial on Particle Filters for Online Nonlinear/non-Gaussian Bayesian Tracking," IEEE Trans. Signal Process., vol. 50, no. 2, pp. 174-188, Feb. 2002.

[56] F. de Ponte Müller, "Survey on Ranging Sensors and Cooperative Techniques for Relative Positioning of Vehicles," Sensors, vol. 17, no. 2, 2017.

[57] OpenStreetMap contributors, "Planet dump retrieved from https://planet.osm.org ," https://www.openstreetmap.org , 2017.

[58] J. Barceló and J. Casas, Dynamic Network Simulation with AIMSUN. Boston, MA: Springer US, 2005, pp. 57-98.

[59] F. Deflorio and L. Castello, "Assessing the Performance of a ChargeWhile-Driving System in Urban Arterial Roads: Insight from a Microsimulation Model," IET Intell. Transp. Syst., vol. 9, no. 5, pp. 505514, Jun. 2015.

[60] P. Hidas, "Modelling Vehicle Interactions in Microscopic Simulation of Merging and Weaving," Transp. Research Part C: Emerging Technologies, vol. 13, no. 1, pp. 37 - 62, Jan. 2005.

[61] SAE, Taxonomy and Definitions for Terms Related to On-Road Motor Vehicle Automated Driving Systems, Jan. 2014. [Online]. Available: https://doi.org/10.4271/J3016_201806

[62] R. Tachet, P. Santi, S. Sobolevsky, L. I. Reyes-Castro, E. Frazzoli, D. Helbing, and C. Ratti, "Revisiting Street Intersections Using SlotBased Systems," PLOS ONE, vol. 11, pp. 1-9, Mar. 2016.
[63] "Intelligent Transport Systems (ITS); Vehicular Communications; Basic Set of Applications; Definitions," ETSI TR 102 638, Jun. 2009.

[64] C. W. Gardiner, Handbook of stochastic methods for physics, chemistry and the natural sciences, 3rd ed., Berlin, 2004, vol. 13.

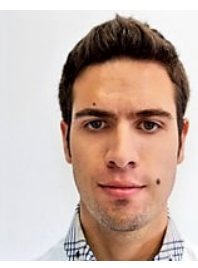

Mattia Brambilla received the B.S. degree (2015) and the M.S. degree (2017) in Telecommunication Engineering from Politecnico di Milano. Currently, he is a Ph.D. student in Information Technology at the Dipartimento di Elettronica, Informazione e Bioingegneria (DEIB) of Politecnico di Milano. His current research interests concern processing techniques for communication and localization in vehicular and industrial environments. He won the best student paper award at the IEEE Statistical Signal Processing Workshop, 2018.

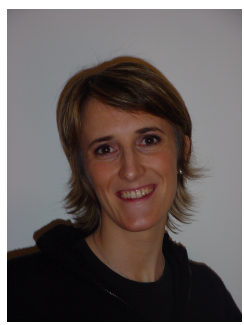

Monica Nicoli received the M.Sc. in Telecommunication Engineering (cum laude) and the Ph.D. in Electronic and Communication Engineering from Politecnico di Milano, in 1998 and 2002, respectively. She was Visiting Researcher with Uppsala University, in 2001. In 2002, she joined as Faculty Member Politecnico di Milano where she is currently Associate Professor. Her research interests are in the area of statistical signal processing and wireless communications, with focus on localization, smart mobility, cooperative and distributed systems for the Internet of Things/Vehicles. She has co-authored over 100 scientific publications. She is recipient of the Marisa Bellisario Award (1999), and corecipient of the Best Paper Awards of the IEEE Statistical Signal Processing Workshop (2018) and the IET ITS Journal (2014). She served as Associate Editor of the EURASIP Journal on Wireless Communications and Networking from 2010 to 2017, and as Lead Guest Editor for the Special Issue on Localization in Mobile Wireless and Sensor Networks in 2011.

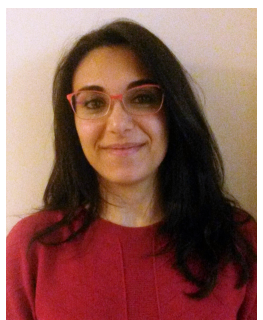

Gloria Soatti received the M.Sc. degree in telecommunication engineering and the Ph.D. degree (cum laude) in information technology from Politecnico di Milano, Italy, in 2013 and 2017, respectively. In 2016 she was a Visiting Researcher with the Chalmers University of Technology, Sweden, and a Post-Doctoral Researcher with the Politecnico di Milano until Feb. 2018. She is now working in Fastweb SpA, located in Milano, Italy, focusing on network functions virtualization and on IP backbone development and test.

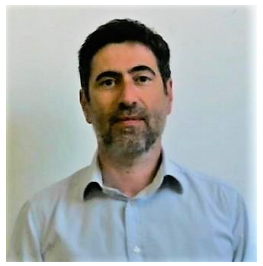

Francesco Deflorio received the M.Sc. degree in Civil Engineering from Politecnico di Bari and Ph.D in Automatics and Information Science in Transportation Systems from Politecnico di Torino, Italy, where he joined as assistant professor in 2006. Currently he is associate professor in Transport System Engineering and his research interests include modeling and applications of traffic and transportation systems, such as dynamic route guidance in road networks, traffic and energy simulation analysis, inover 70 scientific papers. telligent transport systems (ITS). He has co-authored 